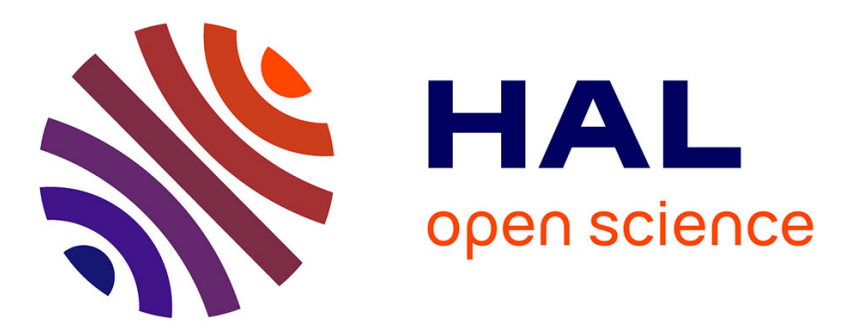

\title{
A semi-implicit electromagnetic FEM-PIC scheme with exact energy and charge conservation
}

Valentin Pagès, Martin Campos Pinto

\section{To cite this version:}

Valentin Pagès, Martin Campos Pinto. A semi-implicit electromagnetic FEM-PIC scheme with exact energy and charge conservation. 2020. hal-02521069v1

\section{HAL Id: hal-02521069 \\ https://hal.sorbonne-universite.fr/hal-02521069v1}

Preprint submitted on 27 Mar 2020 (v1), last revised 17 Sep 2021 (v3)

HAL is a multi-disciplinary open access archive for the deposit and dissemination of scientific research documents, whether they are published or not. The documents may come from teaching and research institutions in France or abroad, or from public or private research centers.
L'archive ouverte pluridisciplinaire HAL, est destinée au dépôt et à la diffusion de documents scientifiques de niveau recherche, publiés ou non, émanant des établissements d'enseignement et de recherche français ou étrangers, des laboratoires publics ou privés. 


\title{
A semi-implicit electromagnetic FEM-PIC scheme with exact energy and charge conservation
}

\author{
Valentin Pagès ${ }^{\mathrm{a}, \mathrm{b}}$, Martin Campos Pinto ${ }^{\mathrm{c}}$ \\ ${ }^{a}$ Sorbonne Université, CNRS, Université de Paris, Laboratoire Jacques-Louis Lions \\ (LJLL), F-75005 Paris, France \\ ${ }^{b}$ Thales AVS/MIS, Computational Electromagnetics, F-78140 Vélizy-Villacoublay, France \\ ${ }^{c}$ Max-Planck-Institut für Plasmaphysik, Boltzmannstraße 2, D-85748 Garching, Germany
}

\begin{abstract}
This article presents a new charge- and energy-conserving semi-implicit method (ChECSIM) for the Vlasov-Maxwell system. The Particle-in-Cell space discretization involves a structure-preserving Finite Element Method for the fields, and the time discretization is an adaption of Lapenta's energy-conserving semiimplicit method (ECSIM) [31, which essentially consists of improving the latter with appropriate charge-conserving current deposition terms and an explicit correction step. As such, the resulting scheme is free of nonlinear iterations, and it preserves both energy and charge exactly.
\end{abstract}

Keywords: Semi-implicit particle-in-cell, Energy conservation, Charge conservation, Vlasov-Maxwell, Kinetic plasma simulation, Structure-preserving finite elements

\section{Introduction}

The long-time accuracy of numerical simulations usually relies on the fact that fundamental invariants of the physical system, such as its total energy, are well preserved at the discrete level. In the case of electromagnetic particle solvers, it has been long recognized that Gauss's laws also represent key invariants [7, 32, 46], with spurious virtual charges building up when they are not preserved by the numerical method, see e.g. 4, 42, 11. At the continuous level, the preservation of Gauss's laws by the time-dependent Maxwell-Vlasov equations hinges on two properties: (i) the charge and current densities satisfy a continuity equation, and (ii) the divergence of a curl is always zero. On a larger perspective, the key role played by Gauss's laws in the long-time stability of Maxwell's equations is now well understood in the scope of their de Rham structure [8, 26, 25, 13].

Email addresses: pagesv@ljll.math.upmc.fr (Valentin Pagès), valentin.pages@thalesgroup.com (Valentin Pagès), martin.campos-pinto@ipp.mpg.de (Martin Campos Pinto)

Preprint submitted to Journal of Computational Physics

March 23, 2020 
In Particle-in-Cell (PIC) methods [27, 5] where Maxwell solvers are coupled to particle approximate sources, a variety of charge- (that is, Gauss-) preserving solvers have been proposed to adress this issue. They may be divided in two categories: field correction methods, where the electromagnetic field is modified to eliminate its deficiency in Gauss's laws [7, 37, 30, 32, and conservative deposition methods, where the current density is computed in a way that preserves a proper continuity equation at the discrete level [46, 21, 19, 45, 11]. Similarly as on the continuous level, these methods also require that the Maxwell solver preserves some essential features of the underlying de Rham structure. For this reason they may be connected to the larger class of structure-preserving methods, which were extensively studied in the last decades : see e.g. [26, 35, 1, 2] for the general properties of these methods, and 9, 14, 29, 28, 38, 36, 43, for their applications in particular problems from plasma physics or electrical engineering.

Recently, several PIC codes have been proposed that preserve exactly the total energy of the discrete system. In [33, Markidis and Lapenta have proposed an energy-conserving method based on an implicit time discretization. The resulting fully nonlinear time steps are solved by an iterative Newton Krylov scheme, and a particle enslavement technique is proposed to reformulate the nonlinear problems on the fields only. In this method the Gauss laws are satisfied approximately, by adding field correction terms as pseudo-currents in the nonlinear problems. In a related method [15], Chen, Chacón and Barnes have taken this approach a step further by taking advantage of the particle enslavement principle to deposit the current with a charge-conserving algorithm, which allows to preserve exactly the Gauss laws even when the iterative Newton scheme is not applied until convergence. At this point we may remind that the classical, explicit leap-frog time stepping 27 coupled with a charge-conserving current deposition [19, 11] allows to preserve the Gauss laws, but it only preserves a modified discrete energy. As a consequence of its explicit nature, stable runs require an explicit CFL constraint which limits the time steps to approximately the cell propagation time of a light wave. In contrast, energy conserving schemes enjoy the possibility of using much larger time steps. However in both methods above the implicit steps are fully nonlinear.

This limitation is removed in the recent Energy-Conserving Semi-Implicit Method (ECSIM) proposed by Lapenta 31. In this novel scheme, the particle enslavement principle is cleverly applied at the very level of the time stepping in such a way that the implicit problems are not only reduced to the field unknowns, but they also become linear. A drawback of this semi-implicit time stepping is that it is a priori not compatible with a charge-conserving current deposition algorithm. Indeed the latter involves time-averages on the particle trajectories, which cannot be expressed as a linear function of the electric field.

To address this issue, a few methods have been devised: in [16] the ECSIM scheme is completed by a first order correction on the particle positions, based on a linearization of the particle shapes. A different approach is followed in [28], where the authors consider the general framework of geometric (hamiltonian) and structure-preserving FEM-PIC methods [29]. There a time stepping is 
proposed which preserves exactly the energy and the Gauss laws, albeit at the cost of a new nonlinear problem to be solved on the fields.

In this article we propose a new method where the semi-implicit time stepping is free of any nonlinear iterations, and where both the energy and Gauss laws are preserved exactly. Our strategy essentially consists of first endowing Lapenta's ECSIM scheme [31 with a semi-conservative current deposition, and of adding an explicit correction to complete the time step with a fully charge-conserving deposition. A velocity update is eventually performed in order to restore the energy conservation of the original scheme. As a result, the computational cost of ChECSIM is close to that of ECSIM with an additional conservative current deposition. similar to what is done in the explicit chargeconserving leap-frog scheme [11. Moreover it is naturally formulated in the general framework of structure-preserving finite elements, as in [14, 40].

This framework is important as it also allows unstructured meshes on complex geometries, with possible local grid adaptations. Indeed, one important field of applications is the industrial context of vacuum electronic devices, where designers are interested in performing numerous long-time simulations within a rather short time-frame. Fast solvers usually rely on simplified models, such as separating the representation of the fields in the beam area in resonant Klystron cavities, see e.g. [17, 22. Nevertheless, in the ever more challenging improvement of the radio-frequency power sources, simulations based on reduced models have shown difficulties to capture troublemaking phenomena that impede the development of new components, and consequently the demand for solvers of the complete time-dependent Maxwell-Vlasov model has arisen. To this end, stable schemes emancipating from CFL constraints and having a complexity that remains affordable for short runtime are attractive, provided that they are robust enough to show long-time accuracy.

The article is organized as follows. After recalling the model in Section 2 we summarize in Section 3 the principles of structure-preserving FEM-PIC spatial discretizations. Once this is settled, we provide in Section 4 a comparative description of the two algorithms that we use as reference FEM-PIC schemes, namely the explicit, charge-conserving leap-frog (CCLF) scheme [11] and the ECSIM scheme of Lapenta [31, reformulated in the framework of finite elements. With these building blocks at hand we can introduce the ChECSIM scheme in Section 5. The guess step is described in Section 5.1 as a semi-conservative modification of the original ECSIM, and the corrective step is presented in Section 5.2. This section is completed by an a priori estimate which shows that the eventual velocity correction is possible under a mild condition on the time step that is linked to the steepness of the electric field, see Proposition 5.4. If necessary, a dynamic adaptation of the time step suffices to fulfill this criterion. In Section 6 we finally perform some numerical experiments on a couple of standard 2D test cases from plasma physics, which confirm the improved stability properties of the proposed method. 


\section{General Framework}

\subsection{The continuous model}

Vlasov-Maxwell's equations are among the most accurate models in plasma physics or in the design of radio-frequency sources. They consist of Vlasov's equation for the charged particles phase-space density,

$$
\partial_{t} f+\boldsymbol{v} \cdot \nabla_{x} f+\frac{q}{m}(\boldsymbol{E}+\boldsymbol{v} \times \boldsymbol{B}) \cdot \nabla_{v} f=0,
$$

(1) eq:vlasov\}

which essentially expresses the transport of particles with elementary charge $q$ and mass $m$ along the electromagnetic Lorentz force, and of Maxwell's equations for the electromagnetic fields. These may also be decomposed in two sets of equations: an evolution system consisting of Ampère and Faraday's equations

$$
\begin{aligned}
\partial_{t} \boldsymbol{E}-c^{2} \operatorname{curl} \boldsymbol{B} & =-\frac{1}{\epsilon_{0}} \boldsymbol{J}, \\
\partial_{t} \boldsymbol{B}+\operatorname{curl} \boldsymbol{E} & =0,
\end{aligned}
$$

and Gauss's laws

$$
\begin{aligned}
\operatorname{div} \boldsymbol{E} & =\frac{1}{\epsilon_{0}} \rho, \\
\operatorname{div} \boldsymbol{B} & =0 .
\end{aligned}
$$

Here, the source terms are the charge and current densities carried by the particles' distribution, defined as the first and second velocity moments of $f$,

$$
\begin{gathered}
\rho=\rho[f]=q \int_{\mathbb{R}^{3}} f \mathrm{~d} \boldsymbol{v}, \\
\boldsymbol{J}=\boldsymbol{J}[f]=q \int_{\mathbb{R}^{3}} \boldsymbol{v} f \mathrm{~d} \boldsymbol{v} .
\end{gathered}
$$

\{eq:part_charge $\}$

It should be noted that the evolution system composed of the Vlasov-AmpèreFaraday equations, namely (1)-(3), defines a unique solution in time starting from an initial one. The Gauss laws (4)-(5) may then be seen as divergence constraints on the fields, and it is an elementary exercice to verify that (i) they will be preserved by any solution to (1)-(3) if the sources satisfy the so-called continuity equation,

$$
\partial_{t} \rho+\operatorname{div} \boldsymbol{J}=0,
$$

(8) $\quad$ eq: continuity $\}$

and (ii) this continuity equation is satisfied by any source of the form (6)-(7), as long as $f$ solves a transport equation of the form (1).

As emphasized in the introduction, another key invariant is the total energy

$$
\mathcal{E}=\frac{\epsilon_{0}\|\boldsymbol{E}\|^{2}}{2}+\frac{\|\boldsymbol{B}\|^{2}}{2 \mu_{0}}+\frac{m}{2} \int|\boldsymbol{v}|^{2} f \mathrm{~d} \boldsymbol{v}
$$

which is preserved by exact solutions in the absence of external contributions. 


\subsection{Discrete FEM-PIC models}

In finite element Particle-In-Cell approximations of the Vlasov-Maxwell system, the electromagnetic field $(\boldsymbol{E}, \boldsymbol{B})$ is represented by a finite element field $\left(\boldsymbol{E}_{h}, \boldsymbol{B}_{h}\right)$, typically a piecewise polynomial on a given mesh of the computational domain $\Omega \subset \mathbb{R}^{3}$, that is determined as the solution to some discrete version of the evolution equations (2)-(3), namely Ampère's and Faraday's equations. This will be specified in Section 3 and below. As for the plasma plase-space density $f$, it is represented by a weighted collection of $N$ numerical particles (here with Dirac masses), of the form

$$
f_{N}(t, \boldsymbol{x}, \boldsymbol{v})=\sum_{k=1}^{N} w_{k} \delta\left(\boldsymbol{x}-\boldsymbol{x}_{k}(t)\right) \delta\left(\boldsymbol{v}-\boldsymbol{v}_{k}(t)\right),
$$

with positions $\boldsymbol{x}_{k}$ and velocities $\boldsymbol{v}_{k}$ that follow discrete Lorentz force trajectories. The coupling of these discrete solutions then consists of specifying (i) how the finite element fields define the Lorentz force at the particle positions, see Section 3.3 and (ii) how the current and charge density sources in the discrete FEM Maxwell system are defined from their particle approximations,

$$
\begin{aligned}
\rho_{N} & :=\int f_{N} \mathrm{~d} \boldsymbol{v}=\sum_{p=1}^{N} q_{k} \delta\left(\boldsymbol{x}-\boldsymbol{x}_{k}(t)\right), \\
\boldsymbol{J}_{N} & :=\int \boldsymbol{v} f_{N} \mathrm{~d} \boldsymbol{v}=\sum_{k=1}^{N} q_{k} \boldsymbol{v}_{k}(t) \delta\left(\boldsymbol{x}-\boldsymbol{x}_{k}(t)\right) .
\end{aligned}
$$

Here $q_{k}=q w_{k}$ is the charge carried by the numerical particle of index $k$, and likewise $m_{k}=m w_{k}$ will denote its mass (for simplicity we consider here a single particle species of charge $q$ and mass $m$ ).

Since FEM-PIC solutions rely only on the time-domain equations (1)-(3), preserving a discrete version of the Gauss laws (4)-(5), as well as a discrete analog to the total energy (9), is clearly a non-straightforward feature to enforce. In particular, we already observe that preserving the Gauss laws will require a particular care in the discretization of the FEM current density source, indeed the latter plays a pivotal role in the charge-conserving coupling between Maxwell and Vlasov equations.

\section{Spatial discretization with structure-preserving finite elements}

In this section we recall the main features of a structure-preserving finite elements discretization of the Maxwell equations, and its coupling with point particles. Similarly as in [34, 43, 36, 14, 29, 38, we follow the general framework of Finite Element Exterior Calculus (FEEC), detailed in [35, 1, 2]. It essentially

relies on finite elements spaces that preserve the de Rham structure of the continuous problem [8. 


\subsection{Mixed weak-form of Maxwell equations}

For simplicity, we consider here that the Maxwell equations are posed on a bounded open domain $\Omega$ of $\mathbb{R}^{3}$ with Lipschitz-continuous boundary supplemented with boundary conditions of metallic type, see e.g. [35,

$$
\boldsymbol{n} \times \boldsymbol{E}=0 \quad \text { on } \quad \partial \Omega
$$

(for boundary conditions of absorbing type, we refer to Appendix A). This leads us to considering the following de Rham sequence,

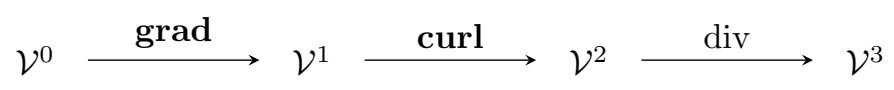

with Hilbert spaces $\mathcal{V}^{0}=H_{0}^{1}(\Omega), \mathcal{V}^{1}=\boldsymbol{H}_{0}(\operatorname{curl}, \Omega), \mathcal{V}^{2}=\boldsymbol{H}_{0}(\operatorname{div}, \Omega)$ and $\mathcal{V}^{3}=L^{2}(\Omega)$, endowed with their standard norms [6, 3].

The first step is to reformulate the time-dependent Maxwell equations in weak form, using only the spaces involved in the sequence (14). Following e.g. [34, a common choice is to integrate by parts in the Ampère equation. Given the metallic boundary condition on the electric field (13), this gives

$$
\begin{aligned}
\left\langle\partial_{t} \boldsymbol{E}(t), \boldsymbol{\phi}^{1}\right\rangle-c^{2}\left\langle\boldsymbol{B}(t), \operatorname{curl} \boldsymbol{\phi}^{1}\right\rangle & =-\frac{1}{\epsilon_{0}}\left\langle\boldsymbol{J}(t), \boldsymbol{\phi}^{1}\right\rangle, & & \forall \boldsymbol{\phi}^{1} \in \mathcal{V}^{1}, \\
\left\langle\partial_{t} \boldsymbol{B}(t), \boldsymbol{\phi}^{2}\right\rangle+\left\langle\operatorname{curl} \boldsymbol{E}(t), \boldsymbol{\phi}^{2}\right\rangle & =0, & & \forall \boldsymbol{\phi}^{2} \in \mathcal{V}^{2}
\end{aligned}
$$

\{eq: var-ampere\}

\{eq:var-faraday $\}$

for all $t \in[0, T]$. Here the bracket $\langle\cdot, \cdot\rangle$ denotes the standard scalar product in $L^{2}(\Omega)$, and the solution $(\boldsymbol{E}, \boldsymbol{B})$ is sought for in the spaces $C^{1}\left([0, T] ; \mathcal{V}^{1}\right) \times$ $C^{1}\left([0, T] ; \mathcal{V}^{2}\right)$. We observe that $\operatorname{curl} \boldsymbol{E} \in C^{0}\left([0, T] ; \mathcal{V}^{2}\right)$, so that 16$]$ amounts to writing the Faraday equation in a strong sense, namely

$$
\partial_{t} \boldsymbol{B}(t)+\operatorname{curl} \boldsymbol{E}(t)=0 \quad \forall t \in[0, T] .
$$

$\{$ eq: strong-faraday $\}$

\subsection{Structure-preserving finite elements}

In order to preserve the de Rham structure at the discrete level, we consider structure-preserving finite element spaces which form a discrete sequence,

$$
V_{h}^{0} \stackrel{\text { grad }}{\longrightarrow} V_{h}^{1} \stackrel{\text { curl }}{\longrightarrow} V_{h}^{2} \stackrel{\text { div }}{\longrightarrow} V_{h}^{3} .
$$

$\{$ deRham_h

As studied in 13, 14, a key feature of such discretization spaces is to provide discrete Gauss laws that allow for a rigorous long-time stability analysis of charge-conserving Maxwell solvers. Moreover, it is known that structurepreserving FEM schemes associated with stable commuting diagrams are free of spurious eigenvalues for the Maxwell evolution operator, see e.g. [26, 1, 12. Several sequences of finite elements may be chosen here. A classical option on unstructured meshes consists in using the Lagrange-Nédélec-Raviart-Thomas sequence [24, 6] studied and generalized in [1, see also [12]. Another option is 
to follow [29] and use the compatible spline spaces defined on tensor-product domains [9, 10, 41], or mimetic spectral elements [23, 39].

Using structure-preserving finite elements we approximate the equations above by a semi-discrete system

$$
\begin{aligned}
\left\langle\partial_{t} \boldsymbol{E}_{h}(t), \boldsymbol{\phi}_{h}^{1}\right\rangle-c^{2}\left\langle\boldsymbol{B}_{h}(t), \operatorname{curl} \boldsymbol{\phi}_{h}^{1}\right\rangle & =-\frac{1}{\epsilon_{0}}\left\langle\boldsymbol{J}_{h}(t), \boldsymbol{\phi}_{h}^{1}\right\rangle, \quad \forall \boldsymbol{\phi}_{h}^{1} \in V_{h}^{1}, \\
\partial_{t} \boldsymbol{B}_{h}(t)+\operatorname{curl} \boldsymbol{E}_{h}(t) & =0
\end{aligned}
$$

again for all $t \in[0, T]$. Here the solution $\left(\boldsymbol{E}_{h}, \boldsymbol{B}_{h}\right)$ is sought for in the spaces $C^{1}\left([0, T] ; V_{h}^{1}\right) \times C^{1}\left([0, T] ; V_{h}^{2}\right)$, and $\boldsymbol{J}_{h}$ is a source current density in $C^{0}\left([0, T] ; V_{h}^{1}\right)$. For the subsequent study of the charge-conservation properties, it will be convenient to rewrite the weak Ampère equation as

$$
\partial_{t} \boldsymbol{E}_{h}-c^{2} \operatorname{curl}_{h} \boldsymbol{B}_{h}=-\frac{1}{\epsilon_{0}} \boldsymbol{J}_{h}
$$

where the discrete operator

$$
\operatorname{curl}_{h}: V_{h}^{2} \rightarrow V_{h}^{1}
$$

is defined as the discrete adjoint of the operator curl : $V_{h}^{1} \rightarrow V_{h}^{2}$, i.e., by the relations

$$
\left\langle\operatorname{curl}_{h} \phi_{h}^{2}, \phi_{h}^{1}\right\rangle=\left\langle\phi_{h}^{2}, \operatorname{curl} \phi_{h}^{1}\right\rangle, \quad \forall \phi_{h}^{1} \in V_{h}^{1} \text { and } \phi_{h}^{2} \in V_{h}^{2} .
$$

Following [13, 14, we know that this discrete functional setting allows to derive long-time stability estimates when the associated discrete Gauss laws, namely

$$
\begin{aligned}
\operatorname{div}_{h} \boldsymbol{E}_{h} & =\frac{1}{\epsilon_{0}} \rho_{h} \\
\operatorname{div} \boldsymbol{B}_{h} & =0
\end{aligned}
$$

are preserved in time. Here the magnetic Gauss law is written in strong form and is obviously preserved since Faraday's equation is also solved in a strong form 20 , whereas 23 involves the discrete weak divergence

$$
\operatorname{div}_{h}: V_{h}^{1} \rightarrow V_{h}^{0}
$$

defined as the adjoint of the operator $-\operatorname{grad}: V_{h}^{0} \rightarrow V_{h}^{1}$, and $\rho_{h} \in V_{h}^{0}$ is defined as the $L^{2}$ projection of the particle charge density (11), that is,

$$
\left\langle\rho_{h}(t), \phi_{h}^{0}\right\rangle=\left\langle\rho_{N}(t), \phi_{h}^{0}\right\rangle=\sum_{k=1}^{N} q_{k} \phi_{h}^{0}\left(\boldsymbol{x}_{k}(t)\right), \quad \forall \phi_{h}^{0} \in V_{h}^{0} .
$$

In particular, the electric Gauss law 23) is equivalently rewritten in a weak finite element sense,

$$
-\left\langle\boldsymbol{E}_{h}, \operatorname{grad} \phi_{h}^{0}\right\rangle=\frac{1}{\epsilon_{0}}\left\langle\rho_{h}, \phi_{h}^{0}\right\rangle, \quad \forall \phi_{h}^{0} \in V_{h}^{0} .
$$


In order to write System (19)- 200 in matrix form, we choose a basis $\Lambda_{i}^{\ell}$, $i=1, \ldots, N_{\ell}$ for each space $V_{h}^{\ell}, \ell=0, \ldots, 3$ and we denote by $\sigma_{i}^{\ell}$ the associated degrees of freedom. The corresponding mass matrices are then

$$
\mathbb{M}_{\ell}=\left(\left\langle\Lambda_{i}^{\ell}, \Lambda_{j}^{\ell}\right\rangle\right)_{1 \leq i, j \leq N_{\ell}},
$$

and we also denote by

$$
\mathbb{C}=\left(\sigma_{i}^{2}\left(\operatorname{curl} \Lambda_{j}^{1}\right)\right)_{1 \leq i \leq N_{2}, 1 \leq j \leq N_{1}}
$$

the $N_{2} \times N_{1}$ matrix of the operator curl $: V_{h}^{1} \rightarrow V_{h}^{2}$. Finally we write the time-dependent field coefficients as column vectors,

$$
\boldsymbol{e}(t)=\left(\sigma_{i}^{1}\left(\boldsymbol{E}_{h}(t)\right)\right)_{1 \leq i \leq N_{1}} \quad \text { and } \quad \boldsymbol{b}(t)=\left(\sigma_{i}^{2}\left(\boldsymbol{B}_{h}(t)\right)\right)_{1 \leq i \leq N_{2}} .
$$

System 19 -20 reads then

$$
\begin{aligned}
\mathbb{M}_{1} \frac{\mathrm{d}}{\mathrm{d} t} \boldsymbol{e}-c^{2}\left(\mathbb{M}_{2} \mathbb{C}\right)^{T} \boldsymbol{b} & =-\frac{1}{\epsilon_{0}} \boldsymbol{j} \\
\frac{\mathrm{d}}{\mathrm{d} t} \boldsymbol{b}+\mathbb{C} \boldsymbol{e} & =0
\end{aligned}
$$

where $\boldsymbol{j}$ contains the moments of the finite element current source, namely

$$
\boldsymbol{j}(t)=\left(\left\langle\boldsymbol{J}_{h}(t), \Lambda_{i}^{1}\right\rangle\right)_{1 \leq i \leq N_{1}} .
$$

\subsection{Energy and charge-conserving coupling with particles}

At the time-continuous level, we consider the same particle-field coupling as done in, e.g., [11. Using discrete point particles, the trajectories read

$$
\left\{\begin{array}{l}
\dot{\boldsymbol{x}}_{k}(t)=\boldsymbol{v}_{k}(t), \\
\dot{\boldsymbol{v}}_{k}(t)=\frac{q_{k}}{m_{k}}\left(\boldsymbol{E}_{h}\left(t, \boldsymbol{x}_{k}(t)\right)+\boldsymbol{v}_{k}(t) \times \boldsymbol{B}_{h}\left(t, \boldsymbol{x}_{k}(t)\right)\right),
\end{array}\right.
$$

and the FEM current source $\boldsymbol{J}_{h} \in V_{h}^{1}$ is defined as the generalized $L^{2}$ projection of the particle current density (12), namely

$$
\left\langle\boldsymbol{J}_{h}(t), \phi_{h}^{1}\right\rangle=\left\langle\boldsymbol{J}_{N}(t), \phi_{h}^{1}\right\rangle=\sum_{k=1}^{N} q_{k} \boldsymbol{v}_{k}(t) \cdot \boldsymbol{\phi}_{h}^{1}\left(\boldsymbol{x}_{k}(t)\right), \quad \forall \boldsymbol{\phi}_{h}^{1} \in V_{h}^{1} .
$$

In the sequel it will be convenient to use a compact notation for the particles positions and velocities,

$$
\boldsymbol{X}=\left(\boldsymbol{x}_{k}\right)_{1 \leq k \leq N} \quad \text { and } \quad \boldsymbol{V}=\left(\boldsymbol{v}_{k}\right)_{1 \leq k \leq N} .
$$


Proposition 3.1. The time-continuous FEM-PIC equations (28)-(31) preserve the discrete energy

$$
\mathcal{E}_{h}(\boldsymbol{V}, \boldsymbol{e}, \boldsymbol{b})=\sum_{k=1}^{N} \frac{m_{k}}{2}\left|\boldsymbol{v}_{k}\right|^{2}+\frac{\epsilon_{0}\left\|\boldsymbol{E}_{h}\right\|^{2}}{2}+\frac{\left\|\boldsymbol{B}_{h}\right\|^{2}}{2 \mu_{0}}
$$

and the discrete Gauss laws (23)-24).

Proof. This result is well known. The conservation of energy is verified by taking the time derivative of $(32)$ and using (30) together with 19$)-(20)$,

$$
\begin{aligned}
\frac{\mathrm{d}}{\mathrm{d} t} \mathcal{E} & =\sum_{k} m_{k} \boldsymbol{v}_{k} \cdot \dot{\boldsymbol{v}}_{k}+\epsilon_{0}\left\langle\boldsymbol{E}_{h}, \partial_{t} \boldsymbol{E}_{h}\right\rangle+\frac{1}{\mu_{0}}\left\langle\boldsymbol{B}_{h}, \partial_{t} \boldsymbol{B}_{h}\right\rangle \\
& =\sum_{k} q_{k} \boldsymbol{v}_{k} \cdot \boldsymbol{E}_{h}\left(\boldsymbol{x}_{k}\right)+c^{2} \epsilon_{0}\left\langle\operatorname{curl} \boldsymbol{E}_{h}, \boldsymbol{B}_{h}\right\rangle-\left\langle\boldsymbol{E}_{h}, \boldsymbol{J}_{h}\right\rangle-\frac{1}{\mu_{0}}\left\langle\boldsymbol{B}_{h}, \operatorname{curl} \boldsymbol{E}_{h}\right\rangle=0
\end{aligned}
$$

where we have used the definition of $\boldsymbol{J}_{h}$ in the last equality, see (31). Turning to the Gauss laws, we first observe that the strong magnetic Gauss law readily follows from the Faraday equation being satisfied in a strong sense. To verify next that the electric Gauss law is preserved, we differentiate in time 26,

$$
\left\langle\partial_{t} \rho_{h}, \phi_{h}^{0}\right\rangle=\sum_{k=1}^{N} q_{k} \boldsymbol{v}_{k} \cdot \operatorname{grad} \phi_{h}^{0}\left(\boldsymbol{x}_{k}\right)=\left\langle\boldsymbol{J}_{h}, \operatorname{grad} \phi_{h}^{0}\right\rangle
$$

which shows that the FE sources satisfy the semi-discrete continuity equation

$$
\partial_{t} \rho_{h}+\operatorname{div}_{h} \boldsymbol{J}_{h}=0
$$

involving the weak divergence operator $\operatorname{div}_{h}$, see (25). Applying this operator to the discrete (weak) Ampere equation (21) yields then

$$
\partial_{t} \operatorname{div}_{h} \boldsymbol{E}_{h}=\operatorname{div}_{h}\left(c^{2} \operatorname{curl}_{h} \boldsymbol{B}_{h}-\frac{1}{\epsilon_{0}} \boldsymbol{J}_{h}\right)=\partial_{t} \frac{1}{\epsilon_{0}} \rho_{h} .
$$

This shows that the discrete Gauss law (23) is preserved.

\section{Two reference FEM-PIC schemes: CCLF and ECSIM}

In this section we describe the two fully discrete FEM-PIC schemes that will serve us as reference methods in this work, corresponding to two different time integrations of the equations (28)-(31). The first one is the charge-conserving leap-frog scheme (CCLF) studied in [11, and the second one is a finite element version of the Energy conserving semi-implicit (ECSIM) scheme of Lapenta 31, similar to the version derived in 40. Although they are naturally expressed on staggered times, we will present them on the same integer time-step $\left[t^{n}, t^{n+1}\right]$ to facilitate comparisons, i.e.

$$
\left(\boldsymbol{X}^{n}, \boldsymbol{V}^{n}, \boldsymbol{e}^{n}, \boldsymbol{b}^{n}\right) \quad \mapsto \quad\left(\boldsymbol{X}^{n+1}, \boldsymbol{V}^{n+1}, \boldsymbol{e}^{n+1}, \boldsymbol{b}^{n+1}\right) .
$$




\subsection{The charge-conserving leap-frog scheme (CCLF)}

The explicit leap-frog scheme [27] consists of integrating both the particle trajectories (30) and the field equations (19)-20) in a staggered fashion. On an integer time-step, this reads

$$
\left\{\begin{aligned}
\boldsymbol{v}_{k}^{n+\frac{1}{2}}-\boldsymbol{v}_{k}^{n} & =\frac{\Delta t}{2} \frac{q_{k}}{m_{k}}\left(\boldsymbol{E}_{h}^{n}\left(\boldsymbol{x}_{k}^{n}\right)+\boldsymbol{v}_{k}^{n} \times \boldsymbol{B}_{h}^{n}\left(\boldsymbol{x}_{k}^{n}\right)\right), \\
\boldsymbol{x}_{k}^{n+1}-\boldsymbol{x}_{k}^{n} & =\Delta t \boldsymbol{v}_{k}^{n+\frac{1}{2}} \\
\boldsymbol{v}_{k}^{n+1}-\boldsymbol{v}_{k}^{n+\frac{1}{2}} & =\frac{\Delta t}{2} \frac{q_{k}}{m_{k}}\left(\boldsymbol{E}_{h}^{n+1}\left(\boldsymbol{x}_{k}^{n+1}\right)+\boldsymbol{v}_{k}^{n+1} \times \boldsymbol{B}_{h}^{n+1}\left(\boldsymbol{x}_{k}^{n+1}\right)\right),
\end{aligned}\right.
$$

for the particle trajectories (note that $\boldsymbol{v}_{k}^{n}=\frac{1}{2}\left(\boldsymbol{v}_{k}^{n+\frac{1}{2}}+\boldsymbol{v}_{k}^{n-\frac{1}{2}}\right)$ ), and

$$
\left\{\begin{aligned}
\boldsymbol{b}^{n+\frac{1}{2}}-\boldsymbol{b}^{n} & =-\frac{\Delta t}{2} \mathbb{C} \boldsymbol{e}^{n} \\
\mathbb{M}_{1}\left(\boldsymbol{e}^{n+1}-\boldsymbol{e}^{n}\right) & =\Delta t\left(c^{2}\left(\mathbb{M}_{2} \mathbb{C}\right)^{T} \boldsymbol{b}^{n+\frac{1}{2}}-\frac{1}{\epsilon_{0}} \boldsymbol{j}^{n+\frac{1}{2}}\right) \\
\boldsymbol{b}^{n+1}-\boldsymbol{b}^{n+\frac{1}{2}} & =-\frac{\Delta t}{2} \mathbb{C} \boldsymbol{e}^{n+1}
\end{aligned}\right.
$$

for the Maxwell equations. Following [19, 11] we consider here a time averaged current source, namely

$$
\boldsymbol{j}^{n+\frac{1}{2}}=\left(\sum_{k} q_{k} \boldsymbol{v}_{k}^{n+\frac{1}{2}} \cdot\left\{\Lambda_{i}^{1}\right\}_{k}^{n}\right)_{1 \leq i \leq N_{1}}
$$

where the curly brackets denote time averages over interpolated trajectories,

$$
\{g\}_{k}^{n}:=\frac{1}{\Delta t} \int_{t^{n}}^{t^{n+1}} g\left(\boldsymbol{x}_{k}(t)\right) \mathrm{d} t \quad \text { with } \quad \boldsymbol{x}_{k}(t)=\boldsymbol{x}_{k}^{n}+\left(t-t^{n}\right) \boldsymbol{v}_{k}^{n+\frac{1}{2}} .
$$

$\{$ traj_av

We observe that (35) may be expressed in terms of the finite element fields and operators defined in Section 3.2 as

$$
\left\{\begin{aligned}
\boldsymbol{B}_{h}^{n+\frac{1}{2}}-\boldsymbol{B}_{h}^{n} & =-\frac{\Delta t}{2} \operatorname{curl} \boldsymbol{E}_{h}^{n} \\
\boldsymbol{E}_{h}^{n+1}-\boldsymbol{E}_{h}^{n} & =c^{2} \Delta t \operatorname{curl}_{h} \boldsymbol{B}_{h}^{n+\frac{1}{2}}-\frac{\Delta t}{\epsilon_{0}} \boldsymbol{J}_{h}^{n+\frac{1}{2}} \\
\boldsymbol{B}_{h}^{n+1}-\boldsymbol{B}_{h}^{n+\frac{1}{2}} & =-\frac{\Delta t}{2} \operatorname{curl} \boldsymbol{E}_{h}^{n+1}
\end{aligned}\right.
$$

with a FEM current source $\boldsymbol{J}_{h} \in V_{h}^{1}$ defined as

$$
\left\langle\boldsymbol{J}_{h}^{n+\frac{1}{2}}, \phi_{h}^{1}\right\rangle=\sum_{k} q_{k} \boldsymbol{v}_{k}^{n+\frac{1}{2}} \cdot\left\{\phi_{h}^{1}\right\}_{k}^{n}, \quad \forall \phi_{h}^{1} \in V_{h}^{1} .
$$

$\{$ eq:cc_current $\}$

An important property of this method is that it preserves exactly the proper discrete Gauss laws, see [11, 14]. 
Proposition 4.1. The CCLF scheme (34)-(35) preserves the discrete Gauss laws (23)-24) evaluated at the discrete times, namely

$$
\begin{aligned}
\operatorname{div}_{h} \boldsymbol{E}_{h}^{n} & =\frac{1}{\epsilon_{0}} \rho_{h}^{n} \\
\operatorname{div} B_{h}^{n} & =0
\end{aligned}
$$

with a FEM charge density defined as the instant value (26), i.e., $\rho_{h}^{n}:=\rho_{h}\left(t^{n}\right)$.

Proof. The argument is the same as for the time continuous result (Prop. 3.1), the key ingredient being that the time-averaged current $(38)$ naturally leads to a proper discrete continuity equation. Indeed the interpolated trajectories (36) have a constant velocity over the time-step $\left[t^{n}, t^{n+1}\right]$, so that the above current density corresponds to

$$
\boldsymbol{J}_{h}^{n+\frac{1}{2}}=\frac{1}{\Delta t} \int_{t^{n}}^{t^{n+1}} \boldsymbol{J}_{h}(t) \mathrm{d} t
$$

where the time-continuous current is defined as in (31). In particular, a time integration of 33 readily yields

$$
\frac{1}{\Delta t}\left(\rho_{h}^{n+1}-\rho_{h}^{n}\right)+\operatorname{div}_{h} \boldsymbol{J}_{h}^{n+\frac{1}{2}}=0,
$$

\{conteq-n\}

and applying the discrete $\operatorname{div}_{h}$ operator to the FEM Ampere equation (37) shows that the discrete Gauss law (39) is indeed preserved. As for 40 , it readily follows from the strong form of the discrete Faraday equation in (37).

\subsection{Energy-Conserving Semi-Implicit Method (ECSIM)}

As is well known, one drawback of the above leap-frog scheme is that it does not preserve the energy of the discrete solution,

$$
\mathcal{E}_{h}\left(\boldsymbol{V}^{n}, \boldsymbol{e}^{n}, \boldsymbol{b}^{n}\right)=\sum_{k} \frac{m_{k}}{2}\left|\boldsymbol{v}_{k}^{n}\right|^{2}+\frac{\epsilon_{0}\left\|\boldsymbol{E}_{h}^{n}\right\|^{2}}{2}+\frac{\left\|\boldsymbol{B}_{h}^{n}\right\|^{2}}{2 \mu_{0}},
$$

$\left\{\mathrm{CE} \_\mathrm{hn}\right\}$

one obvious reason being that even in the source-free case, the leap-frog Maxwell scheme requires a CFL time-stepping condition for $L^{2}$ stability, see e.g. 44]. As recalled in the introduction, a time scheme that exactly preserves energy at the cost of a linear implicitation in the Maxwell solver has been recently proposed by Lapenta [31. Here we describe this energy-conserving semi-implicit method (ECSIM) in our FEM setting. The particle pusher reads

$$
\left\{\begin{aligned}
\boldsymbol{x}_{k}^{n+\frac{1}{2}}-\boldsymbol{x}_{k}^{n} & =\frac{\Delta t}{2} \boldsymbol{v}_{k}^{n}, \\
\boldsymbol{v}_{k}^{n+1}-\boldsymbol{v}_{k}^{n} & =\Delta t \frac{q_{k}}{m_{k}}\left(\boldsymbol{E}_{h}^{n+\frac{1}{2}}\left(\boldsymbol{x}_{k}^{n+\frac{1}{2}}\right)+\boldsymbol{v}_{k}^{n+\frac{1}{2}} \times \boldsymbol{B}_{h}^{n}\left(\boldsymbol{x}_{k}^{n+\frac{1}{2}}\right)\right), \\
\boldsymbol{x}_{k}^{n+1}-\boldsymbol{x}_{k}^{n+\frac{1}{2}} & =\frac{\Delta t}{2} \boldsymbol{v}_{k}^{n+1}
\end{aligned}\right.
$$


with short-hand notations

$$
\boldsymbol{v}_{k}^{n+\frac{1}{2}}:=\frac{1}{2}\left(\boldsymbol{v}_{k}^{n}+\boldsymbol{v}_{k}^{n+1}\right) \quad \text { and } \quad \boldsymbol{E}_{h}^{n+\frac{1}{2}}:=\frac{1}{2}\left(\boldsymbol{E}_{h}^{n}+\boldsymbol{E}_{h}^{n+1}\right),
$$

and the discrete Maxwell solver takes the following implicit form

$$
\left\{\begin{aligned}
\mathbb{M}_{1}\left(\boldsymbol{e}^{n+1}-\boldsymbol{e}^{n}\right) & =\Delta t\left(c^{2}\left(\mathbb{M}_{2} \mathbb{C}\right)^{T} \boldsymbol{b}^{n+\frac{1}{2}}-\frac{1}{\epsilon_{0}} \boldsymbol{j}^{n+\frac{1}{2}}\right), \\
\boldsymbol{b}^{n+1}-\boldsymbol{b}^{n} & =-\Delta t \mathbb{C} e^{n+\frac{1}{2}}
\end{aligned}\right.
$$

$\{$ Max-ecsim $\}$

with $\boldsymbol{e}^{n+\frac{1}{2}}:=\frac{1}{2}\left(\boldsymbol{e}^{n}+\boldsymbol{e}^{n+1}\right), \boldsymbol{b}^{n+\frac{1}{2}}:=\frac{1}{2}\left(\boldsymbol{b}^{n}+\boldsymbol{b}^{n+1}\right)$, and a discrete current now defined through point values at the particle positions,

$$
\boldsymbol{j}^{n+\frac{1}{2}}=\left(\sum_{k} q_{k} \boldsymbol{v}_{k}^{n+\frac{1}{2}} \cdot \Lambda_{i}^{1}\left(\boldsymbol{x}_{k}^{n+\frac{1}{2}}\right)\right)_{1 \leq i \leq N_{1}}
$$

$\{\mathrm{bj}-\mathrm{ecsim}\}$

As will be verified below, this scheme preserves exactly the total energy 42 . One apparent difficulty is that, as the current source 45 involves the unknown velocities $\boldsymbol{v}_{k}^{n+1}$, it is a priori fully implicit. However, just as its original FD version [31, it has the remarkable property that it can be decomposed into a fully explicit particle pusher and a linearly-implicit field solver. In order to see it we rewrite the velocity kick in 43 as

$$
\boldsymbol{v}_{k}^{n+\frac{1}{2}}=\frac{1}{2}\left(\boldsymbol{v}_{k}^{n}+\boldsymbol{v}_{k}^{n+1}\right)=\boldsymbol{v}_{k}^{n}+\frac{q_{k} \Delta t}{2 m_{k}}\left(\boldsymbol{E}_{h}^{n+\frac{1}{2}}\left(\boldsymbol{x}_{k}^{n+\frac{1}{2}}\right)+\mathbb{B}_{k}^{n} \boldsymbol{v}_{k}^{n+\frac{1}{2}}\right),
$$

where the matrix

$$
\mathbb{B}_{k}^{n}:=\left(\begin{array}{ccc}
0 & B_{k, 3}^{n} & -B_{k, 2}^{n} \\
-B_{k, 3}^{n} & 0 & B_{k, 1}^{n} \\
B_{k, 2}^{n} & -B_{k, 1}^{n} & 0
\end{array}\right) \quad \text { with } \quad \boldsymbol{B}_{k}^{n}:=\boldsymbol{B}_{h}^{n}\left(\boldsymbol{x}_{k}^{n+\frac{1}{2}}\right)
$$

is such that $\mathbb{B}_{k}^{n} \boldsymbol{v}=\boldsymbol{v} \times \boldsymbol{B}_{h}^{n}\left(\boldsymbol{x}_{k}^{n+\frac{1}{2}}\right)$ for all $\boldsymbol{v} \in \mathbb{R}^{3}$. Denoting $\eta_{k}=\frac{q_{k} \Delta t}{2 m_{k}}$ and

$$
\begin{aligned}
\mathbb{A}_{k}^{n} & :=\left(\mathbb{I}-\eta_{k} \mathbb{B}_{k}^{n}\right)^{-1} \\
& =\frac{1}{1+|\boldsymbol{\beta}|^{2}}\left(\begin{array}{ccc}
1+\beta_{1}^{2} & -\beta_{3}+\beta_{1} \beta_{2} & \beta_{2}+\beta_{1} \beta_{3} \\
\beta_{3}+\beta_{1} \beta_{2} & 1+\beta_{2}^{2} & -\beta_{1}+\beta_{2} \beta_{3} \\
-\beta_{2}+\beta_{1} \beta_{3} & \beta_{1}+\beta_{2} \beta_{3} & 1+\beta_{3}^{2}
\end{array}\right)
\end{aligned}
$$

here with $\boldsymbol{\beta}:=\eta_{k} \boldsymbol{B}_{h}^{n}\left(\boldsymbol{x}_{k}^{n+\frac{1}{2}}\right)$ as a short-hand notation, this gives

$$
\boldsymbol{v}_{k}^{n+\frac{1}{2}}=\mathbb{A}_{k}^{n}\left(\boldsymbol{v}_{k}^{n}+\eta_{k} \boldsymbol{E}_{h}^{n+\frac{1}{2}}\left(\boldsymbol{x}_{k}^{n+\frac{1}{2}}\right)\right)
$$

which allows us to decompose the fully implicit current 45 in two parts,

$$
\boldsymbol{j}^{n+\frac{1}{2}}=\boldsymbol{j}_{\mathrm{exp}}^{n}+\boldsymbol{j}_{\mathrm{imp}}^{n}\left[\boldsymbol{E}_{h}^{n+\frac{1}{2}}\right]
$$


with explicit and implicit parts defined by the moment arrays

$$
\left\{\begin{aligned}
\boldsymbol{j}_{\exp }^{n}=\left(\sum_{k} q_{k} \mathbb{A}_{k}^{n} \boldsymbol{v}_{k}^{n} \cdot \Lambda_{i}^{1}\left(\boldsymbol{x}^{n+\frac{1}{2}}\right)\right)_{1 \leq i \leq N_{1}}, \\
\boldsymbol{j}_{\mathrm{imp}}^{n}\left[\boldsymbol{E}_{h}^{n+\frac{1}{2}}\right]=\frac{\Delta t}{2}\left(\sum_{k} \frac{q_{k}^{2}}{m_{k}}\left(\mathbb{A}_{k}^{n} \boldsymbol{E}_{h}^{n+\frac{1}{2}}\left(\boldsymbol{x}_{k}^{n+\frac{1}{2}}\right)\right) \cdot \Lambda_{i}^{1}\left(\boldsymbol{x}^{n+\frac{1}{2}}\right)\right)_{1 \leq i \leq N_{1}} \\
=\frac{\Delta t}{2} \mathbb{L}^{n}\left(\frac{\boldsymbol{e}^{n}+\boldsymbol{e}^{n+1}}{2}\right) .
\end{aligned}\right.
$$

Here we have introduced a finite element "Lapenta" matrix [31],

$$
\mathbb{L}^{n}:=\left(\sum_{k} \frac{q_{k}^{2}}{m_{k}} \mathbb{A}_{k}^{n} \Lambda_{j}^{1}\left(\boldsymbol{x}_{k}^{n+\frac{1}{2}}\right) \cdot \Lambda_{i}^{1}\left(\boldsymbol{x}_{k}^{n+\frac{1}{2}}\right)\right)_{1 \leq i, j \leq N_{1}} .
$$

Using (47) and (48) we then rewrite the discrete Maxwell system 444) as

$$
\begin{aligned}
& \left(\begin{array}{cc}
\mathbb{M}_{1}+\frac{\Delta t^{2}}{4 \epsilon_{0}} \mathbb{L}^{n} & -\frac{c^{2} \Delta t}{2}\left(\mathbb{M}_{2} \mathbb{C}\right)^{T} \\
\frac{\Delta t}{2} \mathbb{C} & \mathbb{I}
\end{array}\right)\left(\begin{array}{l}
\boldsymbol{e}^{n+1} \\
\boldsymbol{b}^{n+1}
\end{array}\right) \\
& \quad=\left(\begin{array}{cc}
\mathbb{M}_{1}-\frac{\Delta t^{2}}{4 \epsilon_{0}} \mathbb{L}^{n} & \frac{c^{2} \Delta t}{2}\left(\mathbb{M}_{2} \mathbb{C}\right)^{T} \\
-\frac{\Delta t}{2} \mathbb{C} & \mathbb{I}
\end{array}\right)\left(\begin{array}{l}
\boldsymbol{e}^{n} \\
\boldsymbol{b}^{n}
\end{array}\right)-\frac{\Delta t}{\epsilon_{0}}\left(\begin{array}{c}
\boldsymbol{j}_{\exp }^{n} \\
0
\end{array}\right)
\end{aligned}
$$

and summarize the above steps as follows.

Proposition 4.2. The ECSIM scheme 430-45 is equivalently performed by the following steps:

(i) push the particles between $t^{n}$ and $t^{n+\frac{1}{2}}$ as in (43),

(ii) compute the explicit current term 48) and the Lapenta matrix 49],

(iii) solve the linearly-implicit Maxwell system [50,

(iv) update the velocities as in (43),

(v) push the particles between $t^{n+\frac{1}{2}}$ and $t^{n+1}$ as in 43 .

As claimed above, this scheme enjoys an exact energy conservation property.

Proposition 4.3. The ECSIM scheme (43)- 45 preserves the discrete energy 42 .

Proof. In terms of finite element fields, the Maxwell solver (44) reads :

$$
\left\{\begin{aligned}
\left\langle\boldsymbol{E}_{h}^{n+1}-\boldsymbol{E}_{h}^{n}, \boldsymbol{\phi}_{h}^{1}\right\rangle & =c^{2} \Delta t\left\langle\boldsymbol{B}_{h}^{n+\frac{1}{2}}, \operatorname{curl} \phi_{h}^{1}\right\rangle-\frac{\Delta t}{\epsilon_{0}}\left\langle\boldsymbol{J}_{h}^{n+\frac{1}{2}}, \phi_{h}^{1}\right\rangle, \quad \forall \phi_{h}^{1} \in V_{h}^{1}, \\
\boldsymbol{B}_{h}^{n+1}-\boldsymbol{B}_{h}^{n} & =-\Delta t \operatorname{curl} \boldsymbol{E}_{h}^{n+\frac{1}{2}},
\end{aligned}\right.
$$

$\{$ Max-fem-ecsim $\}$ 
with a current source defined by its moments :

$$
\left\langle\boldsymbol{J}_{h}^{n+\frac{1}{2}}, \phi_{h}^{1}\right\rangle=q \boldsymbol{v}_{k}^{n+\frac{1}{2}} \cdot \phi_{h}^{1}\left(\boldsymbol{x}_{k}^{n+\frac{1}{2}}\right) \quad \text { for all } \phi_{h}^{1} \in V_{h}^{1} .
$$

The energy variation from time-step $n$ to time-step $n+1$ is then the sum of three contributions:

$$
\begin{aligned}
\frac{\left\|\boldsymbol{B}_{h}^{n+1}\right\|^{2}-\left\|\boldsymbol{B}_{h}^{n}\right\|^{2}}{2 \mu_{0}} & =\frac{1}{\mu_{0}}\left\langle\boldsymbol{B}_{h}^{n+1}-\boldsymbol{B}_{h}^{n}, \boldsymbol{B}_{h}^{n+\frac{1}{2}}\right\rangle \\
& =-\frac{1}{\mu_{0}} \Delta t\left\langle\boldsymbol{B}_{h}^{n+\frac{1}{2}}, \operatorname{curl} \boldsymbol{E}_{h}^{n+\frac{1}{2}}\right\rangle \\
\frac{\epsilon_{0}\left(\left\|\boldsymbol{E}_{h}^{n+1}\right\|^{2}-\left\|\boldsymbol{E}_{h}^{n}\right\|^{2}\right)}{2} & =\epsilon_{0}\left\langle\boldsymbol{E}_{h}^{n+1}-\boldsymbol{E}_{h}^{n}, \boldsymbol{E}_{h}^{n+\frac{1}{2}}\right\rangle \\
& =\frac{1}{\mu_{0}} \Delta t\left\langle\boldsymbol{B}_{h}^{n+\frac{1}{2}}, \operatorname{curl} \boldsymbol{E}_{h}^{n+\frac{1}{2}}\right\rangle-\Delta t\left\langle\boldsymbol{J}_{h}^{n+\frac{1}{2}}, \boldsymbol{E}_{h}^{n+\frac{1}{2}}\right\rangle, \\
\sum_{k} \frac{m_{k}}{2}\left(\left|\boldsymbol{v}_{k}^{n+1}\right|^{2}-\left|\boldsymbol{v}_{k}^{n}\right|^{2}\right) & =\sum_{k} m_{k}\left(\boldsymbol{v}_{k}^{n+1}-\boldsymbol{v}_{k}^{n}\right) \cdot \boldsymbol{v}_{k}^{n+\frac{1}{2}} \\
& =\sum_{k} q_{k} \Delta t \boldsymbol{v}_{k}^{n+\frac{1}{2}} \cdot \boldsymbol{E}_{h}^{n+\frac{1}{2}}\left(\boldsymbol{x}_{k}^{n+\frac{1}{2}}\right) \\
& =\Delta t\left\langle\boldsymbol{J}_{h}^{n+\frac{1}{2}}, \boldsymbol{E}_{h}^{n+\frac{1}{2}}\right\rangle .
\end{aligned}
$$

and those three terms cancel out.

We close this section with a study of the solvability of the implicit Maxwell system involved in the finite element ECSIM scheme.

Proposition 4.4. The linear system (50) is always invertible.

Proof. The matrix on the left-hand side of 50 has a block structure

$$
\mathcal{M}=\left(\begin{array}{cc}
\mathbb{M}_{1}+\mathbb{L}^{n} & -\left(\mathbb{M}_{2} \mathbb{C}\right)^{T} \\
\mathbb{C} & \mathbb{I}
\end{array}\right)
$$

where we have removed scalar parameters such as $c$ or $\Delta t$ for the sake of simplicity, since they do not play any role in the argument below. This matrix is invertible if and only if there exists matrices $\mathbb{P}, \mathbb{Q}, \mathbb{R}, \mathbb{S}$ such that

$$
\left(\begin{array}{ll}
\mathbb{P} & \mathbb{Q} \\
\mathbb{R} & \mathbb{S}
\end{array}\right) \mathcal{M}=\left(\begin{array}{ll}
\mathbb{I} & 0 \\
0 & \mathbb{I}
\end{array}\right)
$$

which is equivalent to

$$
\begin{aligned}
\mathbb{Q} & =\mathbb{P}\left(\mathbb{M}_{2} \mathbb{C}\right)^{T}, \\
\mathbb{S} \mathbb{C} & =-\mathbb{R}\left(\mathbb{M}_{1}+\mathbb{L}^{n}\right), \\
\mathbb{P}\left(\mathbb{M}_{1}+\mathbb{L}^{n}+\mathbb{C}^{T} \mathbb{M}_{2} \mathbb{C}\right) & =\mathbb{I}, \\
-\mathbb{R}\left(\mathbb{M}_{1}+\mathbb{L}^{n}+\mathbb{C}^{T} \mathbb{M}_{2} \mathbb{C}\right) & =\mathbb{C} .
\end{aligned}
$$


Hence, the invertiblity of $\mathcal{M}$ is equivalent to the invertibility of the matrices

$$
\mathbb{U}:=\mathbb{M}_{1}+\mathbb{L}^{n} \quad \text { and } \quad \mathbb{V}:=\mathbb{M}_{1}+\mathbb{L}^{n}+\mathbb{C}^{T} \mathbb{M}_{2} \mathbb{C} .
$$

Here the mass matrix $\mathbb{M}_{1}$ is obviously positive definite, since for any function $\boldsymbol{w}_{h} \neq 0$ of $V_{h}^{1}$ with coeficient vector denoted by $\boldsymbol{w}$, one has

$$
\boldsymbol{w}^{T} \mathbb{M}_{1} \boldsymbol{w}=\left\langle\boldsymbol{w}_{h}, \boldsymbol{w}_{h}\right\rangle=\left\|\boldsymbol{w}_{h}\right\|^{2}>0 .
$$

Similarly, $\mathbb{C}^{T} \mathbb{M}_{2} \mathbb{C}$ is positive semi-definite, since

$$
\boldsymbol{w}^{T} \mathbb{C}^{T} \mathbb{M}_{2} \mathbb{C} \boldsymbol{w}=\left\langle\operatorname{curl}_{h} \boldsymbol{w}_{h}, \operatorname{curl}_{h} \boldsymbol{w}_{h}\right\rangle=\left\|\operatorname{curl}_{h} \boldsymbol{w}_{h}\right\|^{2} \geq 0 .
$$

Finally, using (49) and 46) we write

$$
\boldsymbol{w}^{T} \mathbb{L}^{n} \boldsymbol{w}=\sum_{k} \frac{q_{k}^{2}}{m_{k}}\left(\mathbb{A}_{k}^{n} \boldsymbol{w}_{k}^{n}\right) \cdot \boldsymbol{w}_{k}^{n}=\sum_{k} \alpha_{k}\left(\left|\boldsymbol{w}_{k}^{n}\right|^{2}+\left|\boldsymbol{\beta}_{k}^{n} \cdot \boldsymbol{w}_{k}^{n}\right|^{2}\right) \geq 0
$$

with $\alpha_{k}:=\frac{q_{k}^{2}}{m_{k}\left(1+\left|\boldsymbol{\beta}_{k}^{n}\right|^{2}\right)}>0, \boldsymbol{w}_{k}^{n}:=\boldsymbol{w}_{h}\left(\boldsymbol{x}_{k}^{n+\frac{1}{2}}\right)$ and $\boldsymbol{\beta}_{k}^{n}:=\eta_{k} \boldsymbol{B}_{h}^{n}\left(\boldsymbol{x}_{k}^{n+\frac{1}{2}}\right)$. This shows that the Lapenta matrix $\mathbb{L}^{n}$ is also positive semi-definite, so that both $\mathbb{U}$ and $\mathbb{V}$ are positive definite. They are therefore invertible, which proves that the corresponding implicit Maxwell system (50) is well-posed.

\section{ChECSIM: a charge- and energy-conserving semi-implicit scheme}

In the above sections, we have presented two FEM-PIC schemes which preserve either a proper set of discrete Gauss laws, or the discrete energy of the system. However, none of them preserves both quantities. On the other hand, it is possible to take a time-step free from CFL constraint with ECSIM's Maxwell solver but not with CCLF's. We wish to combine all the advantageous properties in a new scheme that keeps ECSIM's low computational cost, that is, a linear-implicit field iteration and an explicit pusher.

In ECSIM, when performing the discrete Ampère's equation (44), it is not possible to straightforwardly apply charge-conserving current deposition of the form of (38) without resorting to a non-linear resolution. Indeed, the trajectory of particles is unknown over the second half of the time-step, since only the first operation in 43 has been performed at this point. To circumvent this obstacle, we propose a prediction/correction two-stepped scheme : in the first step we use a modified ECSIM scheme to compute a temporary solution :

$$
\left(\boldsymbol{X}^{n}, \boldsymbol{V}^{n}, \boldsymbol{e}^{n}, \boldsymbol{b}^{n}\right) \quad \mapsto \quad\left(\boldsymbol{X}^{n+\frac{1}{2}}, \tilde{\boldsymbol{V}}^{n+1}, \tilde{\boldsymbol{e}}^{n+1}, \boldsymbol{b}^{n+1}\right) .
$$

Then we perform a corrective step,

$$
\left(\boldsymbol{X}^{n+\frac{1}{2}}, \tilde{\boldsymbol{V}}^{n+1}, \tilde{\boldsymbol{e}}^{n+1}, \boldsymbol{b}^{n+1}\right) \quad \mapsto \quad\left(\boldsymbol{X}^{n+1}, \boldsymbol{V}^{n+1}, \boldsymbol{e}^{n+1}, \boldsymbol{b}^{n+1}\right),
$$

in such a way that the resulting ChECSIM scheme is both Gauss- and energypreserving. 


\subsection{ChECSIM guess step}

The first step mimics an ECSIM iteration, with a few modifications: the trajectories are now defined by :

$$
\left\{\begin{array}{l}
\boldsymbol{x}_{k}^{n+\frac{1}{2}}-\boldsymbol{x}_{k}^{n}=\frac{\Delta t}{2} \boldsymbol{v}_{k}^{n}, \\
\tilde{\boldsymbol{v}}_{k}^{n+1}-\boldsymbol{v}_{k}^{n}=\Delta t \frac{q_{k}}{m_{k}}\left(\left\{\tilde{\boldsymbol{E}}_{h}^{n+\frac{1}{2}}\right\}_{k}^{n,-}+\tilde{\boldsymbol{v}}_{k}^{n+\frac{1}{2}} \times \boldsymbol{B}_{h}^{n}\left(\boldsymbol{x}_{k}^{n+\frac{1}{2}}\right)\right),
\end{array}\right.
$$

$\{$ traj-checsim-1\}

with short-hand notations :

$$
\tilde{\boldsymbol{v}}_{k}^{n+\frac{1}{2}}:=\frac{1}{2}\left(\boldsymbol{v}_{k}^{n}+\tilde{\boldsymbol{v}}_{k}^{n+1}\right) \quad \text { and } \quad \tilde{\boldsymbol{E}}_{h}^{n+\frac{1}{2}}:=\frac{1}{2}\left(\boldsymbol{E}_{h}^{n}+\tilde{\boldsymbol{E}}_{h}^{n+1}\right),
$$

and the curly brackets with a minus sign denoting a time average over the first half time-step :

$$
\{g\}_{k}^{n,-}:=\frac{2}{\Delta t} \int_{t^{n}}^{t^{n+\frac{1}{2}}} g\left(\boldsymbol{x}_{k}(t)\right) \mathrm{d} t \quad \text { with } \quad \boldsymbol{x}_{k}(t)=\boldsymbol{x}_{k}^{n}+\left(t-t^{n}\right) \boldsymbol{v}_{k}^{n} .
$$

$\{$ traj_av- $\}$

The Maxwell equations become

$$
\left\{\begin{aligned}
\mathbb{M}_{1}\left(\tilde{\boldsymbol{e}}^{n+1}-\boldsymbol{e}^{n}\right) & =\Delta t\left(c^{2}\left(\mathbb{M}_{2} \mathbb{C}\right)^{T} \boldsymbol{b}^{n+\frac{1}{2}}-\frac{1}{\epsilon_{0}} \tilde{\boldsymbol{j}}^{n+\frac{1}{2}}\right), \\
\boldsymbol{b}^{n+1}-\boldsymbol{b}^{n} & =-\Delta t \mathbb{C} \tilde{\boldsymbol{e}}^{n+\frac{1}{2}}
\end{aligned}\right.
$$

$\{$ Max-checsim-1 $\}$

with $\tilde{\boldsymbol{e}}^{n+\frac{1}{2}}:=\frac{1}{2}\left(\boldsymbol{e}^{n}+\tilde{\boldsymbol{e}}^{n+1}\right)$ and $\boldsymbol{b}^{n+\frac{1}{2}}:=\frac{1}{2}\left(\boldsymbol{b}^{n}+\boldsymbol{b}^{n+1}\right)$. Here the current term is

$$
\tilde{\boldsymbol{j}}^{n+\frac{1}{2}}=\left(\sum_{k} q_{k} \tilde{\boldsymbol{v}}_{k}^{n+\frac{1}{2}} \cdot\left\{\Lambda_{i}^{1}\right\}_{k}^{n,-}\right)_{1 \leq i \leq N_{1}} .
$$

We observe that the main difference with ECSIM is that in several places we have used time-averages corresponding to the first half time-step, i.e. $\{\cdot\}_{k}^{n,-}$, instead of point values at $\boldsymbol{x}_{k}^{n+\frac{1}{2}}$. This leaves the energy conservation untouched.

Proposition 5.1. The above guess step preserves the discrete energy 42, i.e.,

$$
\mathcal{E}_{h}\left(\tilde{\boldsymbol{V}}^{n}, \tilde{\boldsymbol{e}}^{n}, \boldsymbol{b}^{n}\right)=\mathcal{E}_{h}\left(\boldsymbol{V}^{n}, \boldsymbol{e}^{n}, \boldsymbol{b}^{n}\right) .
$$

Proof. Similarly as in Proposition 4.3 we rewrite the Maxwell solver (60) in terms of finite element fields,

$$
\left\{\begin{aligned}
\left\langle\tilde{\boldsymbol{E}}_{h}^{n+1}-\boldsymbol{E}_{h}^{n}, \boldsymbol{\phi}_{h}^{1}\right\rangle & =c^{2} \Delta t\left\langle\boldsymbol{B}_{h}^{n+\frac{1}{2}}, \operatorname{curl} \phi_{h}^{1}\right\rangle-\Delta t \frac{1}{\epsilon_{0}}\left\langle\tilde{\boldsymbol{J}}_{h}^{n+\frac{1}{2}}, \phi_{h}^{1}\right\rangle, \\
\boldsymbol{B}_{h}^{n+1}-\boldsymbol{B}_{h}^{n} & =-\Delta t \operatorname{curl} \tilde{\boldsymbol{E}}_{h}^{n+\frac{1}{2}}
\end{aligned}\right.
$$

$\{$ Max-fem-checsim $\}$

with a current term now defined as

$$
\left\langle\tilde{\boldsymbol{J}}_{h}^{n+\frac{1}{2}}, \boldsymbol{\phi}_{h}^{1}\right\rangle=\sum_{k} q_{k} \tilde{\boldsymbol{v}}_{k}^{n+\frac{1}{2}} \cdot\left\{\boldsymbol{\phi}_{h}^{1}\right\}_{k}^{n,-}, \quad \text { for all } \boldsymbol{\phi}_{h}^{1} \in V_{h}^{1} .
$$

$\{$ eq:tilde_j\} 
The same computations then apply, the only difference lying in the fact that point values of the electric field are replaced by time-averages over trajectories. For the kinetic energy this gives

$$
\begin{aligned}
\sum_{k} \frac{m_{k}}{2}\left(\left|\tilde{\boldsymbol{v}}_{k}^{n+1}\right|^{2}-\left|\boldsymbol{v}_{k}^{n}\right|^{2}\right) & =\sum_{k} m_{k} \tilde{\boldsymbol{v}}_{k}^{n+\frac{1}{2}} \cdot\left(\tilde{\boldsymbol{v}}_{k}^{n+1}-\boldsymbol{v}_{k}^{n}\right) \\
& =\sum_{k} q_{k} \Delta t \tilde{\boldsymbol{v}}_{k}^{n+\frac{1}{2}} \cdot\left\{\tilde{\boldsymbol{E}}_{h}^{n+\frac{1}{2}}\right\}_{k}^{n,-} \\
& =\Delta t\left\langle\tilde{\boldsymbol{J}}_{h}^{n+\frac{1}{2}}, \tilde{\boldsymbol{E}}_{h}^{n+\frac{1}{2}}\right\rangle
\end{aligned}
$$

and this cancels out the electric energy update, similarly as in Prop. 4.3

As in the ECSIM scheme, the guess step above may be decomposed as an explicit particle pusher and a linearly implicit field solver. To picture that, we first rewrite the discrete Maxwell system (60) as a block-matrix equation,

$$
\begin{aligned}
\left(\begin{array}{cc}
\mathbb{M}_{1} & -\frac{c^{2} \Delta t}{2}\left(\mathbb{M}_{2} \mathbb{C}\right)^{T} \\
\frac{\Delta t}{2} \mathbb{C} & \mathbb{I}
\end{array}\right)\left(\begin{array}{l}
\tilde{e}^{n+1} \\
\boldsymbol{b}^{n+1}
\end{array}\right) & \\
& =\left(\begin{array}{cc}
\mathbb{M}_{1} & \frac{c^{2} \Delta t}{2}\left(\mathbb{M}_{2} \mathbb{C}\right)^{T} \\
-\frac{\Delta t}{2} \mathbb{C} & \mathbb{I}
\end{array}\right)\left(\begin{array}{l}
\boldsymbol{e}^{n} \\
\boldsymbol{b}^{n}
\end{array}\right)-\frac{\Delta t}{\epsilon_{0}}\left(\begin{array}{c}
\tilde{\boldsymbol{\jmath}}^{n+\frac{1}{2}} \\
0
\end{array}\right),
\end{aligned}
$$

and similarly as for ECSIM, we rewrite the mid-point velocity as

$$
\tilde{\boldsymbol{v}}_{k}^{n+\frac{1}{2}}=\frac{1}{2}\left(\boldsymbol{v}_{k}^{n}+\tilde{\boldsymbol{v}}_{k}^{n+1}\right)=\mathbb{A}_{k}^{n}\left(\boldsymbol{v}_{k}^{n}+\eta_{k}\left\{\tilde{\boldsymbol{E}}_{h}^{n+\frac{1}{2}}\right\}_{k}^{n,-}\right),
$$

with $\eta_{k}=\frac{\Delta t q_{k}}{2 m_{k}}$ and $\mathbb{A}_{k}^{n}=\mathbb{A}_{k}^{n}\left(\boldsymbol{b}^{n}, \boldsymbol{X}^{n+\frac{1}{2}}\right)$ the matrix defined in 466). This allows us to decompose the current in two parts,

$$
\tilde{\boldsymbol{\jmath}}^{n+\frac{1}{2}}=\tilde{\boldsymbol{\jmath}}_{\exp }^{n}+\tilde{\boldsymbol{\jmath}}_{\mathrm{imp}}^{n}\left[\tilde{\boldsymbol{E}}_{h}^{n+\frac{1}{2}}\right],
$$

$\left\{t j_{-} \mathrm{dec}\right\}$

with explicit and implicit parts defined by the moment arrays

$$
\left\{\begin{aligned}
\tilde{\boldsymbol{J}}_{\exp }^{n}=\left(\sum_{k} q_{k}\left(\mathbb{A}_{k}^{n} \boldsymbol{v}_{k}^{n}\right) \cdot\left\{\Lambda_{i}^{1}\right\}_{k}^{n,-}\right)_{1 \leq i \leq N_{1}}, \\
\tilde{\boldsymbol{\jmath}}_{\mathrm{imp}}^{n}\left[\tilde{\boldsymbol{E}}_{h}^{n+\frac{1}{2}}\right]=\frac{\Delta t}{2}\left(\sum_{k} \frac{q_{k}^{2}}{m_{k}}\left(\mathbb{A}_{k}^{n}\left\{\tilde{\boldsymbol{E}}_{h}^{n+\frac{1}{2}}\right\}_{k}^{n,-}\right) \cdot\left\{\Lambda_{i}^{1}\right\}_{k}^{n,-}\right)_{1 \leq i \leq N_{1}} \\
=\frac{\Delta t}{2} \overline{\mathbb{L}}^{n}\left(\frac{\boldsymbol{e}^{n}+\tilde{\boldsymbol{e}}^{n+1}}{2}\right) .
\end{aligned}\right.
$$

and a time-averaged analog to our finite element Lapenta matrix 49,

$$
\overline{\mathbb{L}}^{n}=\left(\sum_{k} \frac{q_{k}^{2}}{m_{k}}\left(\mathbb{A}_{k}^{n}\left\{\Lambda_{j}^{1}\right\}_{k}^{n,-}\right) \cdot\left\{\Lambda_{i}^{1}\right\}_{k}^{n,-}\right)_{1 \leq i, j \leq N_{1}} .
$$


Plugging this decomposition into 68 then gives

$$
\begin{aligned}
& \left(\begin{array}{cc}
\mathbb{M}_{1}+\frac{\Delta t^{2}}{4 \epsilon_{0}} \overline{\mathbb{L}}^{n} & -\frac{c^{2} \Delta t}{2}\left(\mathbb{M}_{2} \mathbb{C}\right)^{T} \\
\frac{\Delta t}{2} \mathbb{C} & \mathbb{I}
\end{array}\right)\left(\begin{array}{l}
\tilde{\boldsymbol{e}}^{n+1} \\
\boldsymbol{b}^{n+1}
\end{array}\right) \\
& =\left(\begin{array}{cc}
\mathbb{M}_{1}-\frac{\Delta t^{2}}{4 \epsilon_{0}} \overline{\mathbb{L}}^{n} & \frac{c^{2} \Delta t}{2}\left(\mathbb{M}_{2} \mathbb{C}\right)^{T} \\
-\frac{\Delta t}{2} \mathbb{C} & \mathbb{I}
\end{array}\right)\left(\begin{array}{c}
\boldsymbol{e}^{n} \\
\boldsymbol{b}^{n}
\end{array}\right)-\frac{\Delta t}{\epsilon_{0}}\left(\begin{array}{c}
\tilde{\boldsymbol{J}}_{\exp }^{n} \\
0
\end{array}\right)
\end{aligned}
$$

which leads us to the following computational steps.

Proposition 5.2. The ChECSIM guess step (58)-(64) is equivalently performed by the following steps:

(i) push the particles between $t^{n}$ and $t^{n+\frac{1}{2}}$ as in (58),

(ii) compute the explicit current term (70) and the new Lapenta matrix (71),

(iii) solve the linearly-implicit Maxwell system (72),

(iv) update the velocities as in 58.

Finally we verify that the arguments used in Proposition 4.4 readily apply to this modified Maxwell system.

Proposition 5.3. The linear system 72 is always invertible.

\subsection{ChECSIM correction step}

As previously remarked, the guess step above does not preserve the Gauss laws, which is due to the fact that the linear decoupling procedure makes it impossible to average over the trajectories over the full time-step. However, since we now have a guess velocity for second half step, we may correct the deposited current so that it becomes charge-conserving. Specifically, decompose the guess current source $(64)$ in two parts,

$$
\tilde{\boldsymbol{J}}_{h}^{n+\frac{1}{2}}=\frac{\boldsymbol{J}_{h}^{n,-}+\tilde{\boldsymbol{J}}_{h}^{n,-}}{2},
$$

\{eq:tilde_j_decomposition $\}$

where $\boldsymbol{J}_{h}^{n,-}$ is a charge-conserving term defined as an average current density,

$$
\left\langle\boldsymbol{J}_{h}^{n,-}, \boldsymbol{\phi}_{h}^{1}\right\rangle=\sum_{k} q_{k} \boldsymbol{v}_{k}^{n} \cdot\left\{\boldsymbol{\phi}_{h}^{1}\right\}_{k}^{n,-}=\frac{2}{\Delta t} \int_{t^{n}}^{t^{n+\frac{1}{2}}}\left\langle\boldsymbol{J}_{h}(t), \phi_{h}^{1}\right\rangle \mathrm{d} t,
$$

$\{$ eq:j_minus $\}$

see (31), 59, and a remainder term

$$
\left\langle\tilde{\boldsymbol{J}}_{h}^{n,-}, \boldsymbol{\phi}_{h}^{1}\right\rangle=\sum_{k} q_{k} \tilde{\boldsymbol{v}}_{k}^{n+1} \cdot\left\{\boldsymbol{\phi}_{h}^{1}\right\}_{k}^{n,-}
$$

$\{$ eq:tilde_j_minus $\}$ 
that is not charge-conserving. The aim of the correction step is then to replace this term by a conserving current for the second half time-step, defined as

$$
\left\langle\boldsymbol{J}_{h}^{n,+}, \boldsymbol{\phi}_{h}^{1}\right\rangle=\sum_{k} q_{k} \tilde{\boldsymbol{v}}_{k}^{n+1} \cdot\left\{\boldsymbol{\phi}_{h}^{1}\right\}_{k}^{n,+},
$$

$\{$ eq:j_plus $\}$

where the curly brackets with a plus sign denote time averages over the second half time-step,

$$
\{g\}_{k}^{n,+}:=\frac{2}{\Delta t} \int_{t^{n+\frac{1}{2}}}^{t^{n+1}} g\left(\boldsymbol{x}_{k}(t)\right) \mathrm{d} t \quad \text { with } \quad \boldsymbol{x}_{k}(t)=\boldsymbol{x}_{k}^{n+\frac{1}{2}}+\left(t-t^{n+\frac{1}{2}}\right) \tilde{\boldsymbol{v}}_{k}^{n+1} .
$$

$\{$ traj_av $\}$

The equations are then as follows.

$$
\left\{\begin{aligned}
\boldsymbol{x}_{k}^{n+1}-\boldsymbol{x}_{k}^{n+\frac{1}{2}} & =\frac{\Delta t}{2} \tilde{\boldsymbol{v}}_{k}^{n+1} \\
\mathbb{M}_{1}\left(\boldsymbol{e}^{n+1}-\tilde{\boldsymbol{e}}^{n+1}\right) & =-\frac{\Delta t}{2 \epsilon_{0}}\left(\boldsymbol{j}^{n,+}-\tilde{\boldsymbol{\jmath}}^{n,-}\right), \\
\boldsymbol{v}_{k}^{n+1} & =\lambda \tilde{\boldsymbol{v}}_{k}^{n+1}
\end{aligned}\right.
$$

$\{$ corr-checsim $\}$

Here the current sources correspond to the moments of 75 and 76, , namely

$$
\left(\boldsymbol{j}^{n,+}-\tilde{\boldsymbol{j}}^{n,-}\right)=\left(\sum_{k} q_{k} \tilde{\boldsymbol{v}}_{k}^{n+1} \cdot\left(\left\{\Lambda_{i}^{1}\right\}_{k}^{n,+}-\left\{\Lambda_{i}^{1}\right\}_{k}^{n,-}\right)\right)_{1 \leq i \leq N_{1}},
$$

and $\lambda>0$ is a correction parameter given by the requirement that

$$
\frac{\epsilon_{0}}{2}\left(\left\|\boldsymbol{E}_{h}^{n+1}\right\|^{2}-\left\|\tilde{\boldsymbol{E}}_{h}^{n+1}\right\|^{2}\right)=\sum_{k} \frac{m_{k}}{2}\left(\left|\tilde{\boldsymbol{v}}_{k}^{n+1}\right|^{2}-\left|\boldsymbol{v}_{k}^{n+1}\right|^{2}\right)=\left(1-\lambda^{2}\right) \sum_{k} \frac{m_{k}}{2}\left|\tilde{\boldsymbol{v}}_{k}^{n+1}\right|^{2},
$$

which yields

$$
\lambda:=\left(1-\frac{\epsilon_{0}\left(\left\|\boldsymbol{E}_{h}^{n+1}\right\|^{2}-\left\|\tilde{\boldsymbol{E}}_{h}^{n+1}\right\|^{2}\right)}{\sum_{k} m_{k}\left|\tilde{\boldsymbol{v}}_{k}^{n+1}\right|^{2}}\right)^{\frac{1}{2}} .
$$

Below we will give a sufficient condition for this parameter to be well-defined. Assuming this for now, we can state the main properties of our scheme.

Theorem 5.1. The ChECSIM scheme defined by the guess step (58)-(59) and the correction step (78)-(80) preserves the discrete Gauss laws (39)-(40) as well as the discrete energy 42 ).

Proof. Both properties are easily verified. By construction, the final electric field $\boldsymbol{E}_{h}^{n+1}$ satisfies a discrete Ampère equation

$$
\boldsymbol{E}_{h}^{n+1}-\boldsymbol{E}_{h}^{n}=c^{2} \Delta t \operatorname{curl}_{h} \boldsymbol{B}_{h}^{n+\frac{1}{2}}-\Delta t \frac{1}{\epsilon_{0}} \boldsymbol{J}_{h}^{n+\frac{1}{2}}
$$

with a current density corresponding to

$$
\boldsymbol{J}_{h}^{n+\frac{1}{2}}=\tilde{\boldsymbol{J}}_{h}^{n+\frac{1}{2}}+\frac{1}{2}\left(\boldsymbol{J}_{h}^{n,+}-\tilde{\boldsymbol{J}}_{h}^{n,-}\right)=\frac{1}{2}\left(\boldsymbol{J}_{h}^{n,-}+\boldsymbol{J}_{h}^{n,+}\right)=\frac{1}{\Delta t} \int_{t^{n}}^{t^{n+1}} \boldsymbol{J}_{h}(t) \mathrm{d} t
$$


where again, $\boldsymbol{J}_{h}(t)$ is the time-continuous current density defined by $(31)$. Similarly as for the charge-conserving leap-frog scheme (Prop. 4.1), this shows that the discrete sources satisfy a continuity equation (41) and since the discrete Maxwell equations are solved by structure-preserving finite elements, this is enough to show that the Gauss equations are preserved. The energy conservation follows from Prop. 5.1 and the requirement 79.

We now provide a sufficient condition for the existence of a correction parameter $\lambda$. It will be convenient to denote by

$$
\left\|\boldsymbol{v}^{n}\right\|_{m}:=\left(\sum_{k} m_{k}\left|\boldsymbol{v}_{k}\right|^{2}\right)^{\frac{1}{2}}
$$

the weighted $\ell^{2}$ norm involved in the kinetic energy.

Proposition 5.4. Let $\boldsymbol{\gamma}_{k}^{n}:=\frac{q}{m}\left\{\tilde{\boldsymbol{E}}_{h}^{n+\frac{1}{2}}\right\}_{k}^{n,-}$ denote the acceleration terms in the guess step (58). If the time-step is such that

$$
2 \Delta t\left\|\gamma^{n}\right\|_{m} \leq\left\|\boldsymbol{v}^{n}\right\|_{m}
$$

then the field correction satisfies

$$
\epsilon_{0}\left(\left\|\boldsymbol{E}_{h}^{n+1}\right\|^{2}-\left\|\tilde{\boldsymbol{E}}_{h}^{n+1}\right\|^{2}\right) \leq C \Delta t^{2}\left\|\tilde{\boldsymbol{v}}^{n+1}\right\|_{m}^{2}\|\nabla \overline{\boldsymbol{E}}\|_{1, \infty}
$$

with $C=\frac{q}{m}(1+\sqrt{5})$ and

$$
\|\nabla \overline{\boldsymbol{E}}\|_{1, \infty}:=\left(\sum_{i, j}\left\|\partial_{j} \bar{E}_{i}\right\|_{L^{\infty}(\Omega)}^{2}\right)^{\frac{1}{2}} \quad \text { with } \quad \overline{\boldsymbol{E}}:=\frac{\boldsymbol{E}_{h}^{n+1}+\tilde{\boldsymbol{E}}_{h}^{n+1}}{2} .
$$

In particular, the correction step (78)-80 is well defined for $\Delta t$ small enough.

Proof. From (58) we compute

$$
\begin{aligned}
\left\|\boldsymbol{v}^{n}\right\|_{m}^{2}-\left\|\tilde{\boldsymbol{v}}^{n+1}\right\|_{m}^{2} & =-\Delta t \sum_{k} m_{k} \boldsymbol{\gamma}_{k}^{n} \cdot\left(\tilde{\boldsymbol{v}}_{k}^{n+1}+\boldsymbol{v}_{k}^{n}\right) \\
& \leq \Delta t\left\|\boldsymbol{\gamma}^{n}\right\|_{m}\left(\left\|\tilde{\boldsymbol{v}}^{n+1}\right\|_{m}+\left\|\boldsymbol{v}^{n}\right\|_{m}\right) \leq \frac{3}{4}\left\|\boldsymbol{v}^{n}\right\|_{m}^{2}+\frac{1}{4}\left\|\tilde{\boldsymbol{v}}^{n+1}\right\|_{m}^{2}
\end{aligned}
$$

where we have used (81) and Young's inequality in the last equation. This gives a bound for the guess velocities

$$
\left\|\tilde{\boldsymbol{v}}^{n+1}\right\|_{m}^{2} \geq \frac{1}{5}\left\|\boldsymbol{v}^{n}\right\|_{m}^{2}
$$

$\{$ vbound

Integrating next the field correction in $(78)$ against $\overline{\boldsymbol{E}}$ and using the definition 
of the respective current terms yields then

$$
\begin{aligned}
\left\|\boldsymbol{E}_{h}^{n+1}\right\|^{2}-\left\|\tilde{\boldsymbol{E}}_{h}^{n+1}\right\|^{2} & =-\frac{\Delta t}{\epsilon_{0}}\left\langle\boldsymbol{J}_{h}^{n,+}-\tilde{\boldsymbol{J}}_{h}^{n,-}, \overline{\boldsymbol{E}}\right\rangle \\
& =-\frac{\Delta t}{\epsilon_{0}} \sum_{k} q_{k} \tilde{\boldsymbol{v}}_{k}^{n+1} \cdot\left(\{\overline{\boldsymbol{E}}\}_{k}^{n,+}-\{\overline{\boldsymbol{E}}\}_{k}^{n,-}\right) \\
& \leq \frac{\Delta t}{\epsilon_{0}} \sum_{k} q_{k}\left|\tilde{\boldsymbol{v}}_{k}^{n+1} \| \boldsymbol{\delta}_{k}^{n}(\overline{\boldsymbol{E}})\right|
\end{aligned}
$$

where we have set $\boldsymbol{\delta}_{k}^{n}(\overline{\boldsymbol{E}}):=\{\overline{\boldsymbol{E}}\}_{k}^{n,+}-\{\overline{\boldsymbol{E}}\}_{k}^{n,-}$. By definition of the time averages (59) and (77) we next compute that

$$
\begin{aligned}
\frac{\Delta t}{2} \boldsymbol{\delta}_{k}^{n}(\overline{\boldsymbol{E}}) & =\int_{t^{n+\frac{1}{2}}}^{t^{n+1}} \overline{\boldsymbol{E}}\left(\boldsymbol{x}_{k}(t)\right) \mathrm{d} t-\int_{t^{n}}^{t^{n+\frac{1}{2}}} \overline{\boldsymbol{E}}\left(\boldsymbol{x}_{k}(t)\right) \mathrm{d} t \\
& =\int_{t^{n+\frac{1}{2}}}^{t^{n+1}} \overline{\boldsymbol{E}}\left(\boldsymbol{x}_{k}(t)\right)-\overline{\boldsymbol{E}}\left(\boldsymbol{x}_{k}^{n+\frac{1}{2}}\right) \mathrm{d} t+\int_{t^{n}}^{t^{n+\frac{1}{2}}} \overline{\boldsymbol{E}}\left(\boldsymbol{x}_{k}^{n+\frac{1}{2}}\right)-\overline{\boldsymbol{E}}\left(\boldsymbol{x}_{k}(t)\right) \mathrm{d} t .
\end{aligned}
$$

Assuming that the finite element field $\overline{\boldsymbol{E}}$ is Lipschitz, the first term is bounded by

$$
\begin{aligned}
\int_{t^{n+\frac{1}{2}}}^{t^{n+1}} \overline{\boldsymbol{E}}\left(\boldsymbol{x}_{k}(t)\right)-\overline{\boldsymbol{E}}\left(\boldsymbol{x}_{k}^{n+\frac{1}{2}}\right) \mathrm{d} t & =\int_{t^{n+\frac{1}{2}}}^{t^{n+1}} \int_{t^{n+\frac{1}{2}}}^{t} \frac{\mathrm{d}}{\mathrm{d} t}\left\{\overline{\boldsymbol{E}}\left(\boldsymbol{x}_{k}(\tau)\right)\right\} \mathrm{d} \tau \mathrm{d} t \\
& =\int_{t^{n+\frac{1}{2}}}^{t^{n+1}} \int_{t^{n+\frac{1}{2}}}^{t} \tilde{\boldsymbol{v}}_{k}^{n+1} \cdot \nabla \overline{\boldsymbol{E}}\left(\boldsymbol{x}_{k}(\tau)\right) \mathrm{d} \tau \mathrm{d} t \\
& \leq \frac{\Delta t^{2}}{2}\left|\tilde{\boldsymbol{v}}_{k}^{n+1}\right|\|\nabla \overline{\boldsymbol{E}}\|_{1, \infty}
\end{aligned}
$$

and similarly for the second one,

$$
\int_{t^{n}}^{t^{n+\frac{1}{2}}} \overline{\boldsymbol{E}}\left(\boldsymbol{x}_{k}^{n+\frac{1}{2}}\right)-\overline{\boldsymbol{E}}\left(\boldsymbol{x}_{k}(t)\right) \mathrm{d} t \leq \frac{\Delta t^{2}}{2}\left|\boldsymbol{v}_{k}^{n}\right|\|\nabla \overline{\boldsymbol{E}}\|_{1, \infty} .
$$

The net electric energy variation induced by the correction 78 reads then :

$$
\begin{aligned}
\left\|\boldsymbol{E}_{h}^{n+1}\right\|^{2}-\left\|\tilde{\boldsymbol{E}}_{h}^{n+1}\right\|^{2} & \leq \frac{\Delta t^{2}}{\epsilon_{0}}\left(\sum_{k} q_{k}\left(\left|\tilde{\boldsymbol{v}}_{k}^{n+1}\right|^{2}+\left|\tilde{\boldsymbol{v}}_{k}^{n+1} \| \boldsymbol{v}_{k}^{n}\right|\right)\right)\|\nabla \overline{\boldsymbol{E}}\|_{1, \infty} \\
& \leq \frac{\Delta t^{2}}{\epsilon_{0}} \frac{q}{m}\left\|\tilde{\boldsymbol{v}}^{n+1}\right\|_{m}\left(\left\|\tilde{\boldsymbol{v}}^{n+1}\right\|_{m}+\left\|\boldsymbol{v}^{n}\right\|_{m}\right)\|\nabla \overline{\boldsymbol{E}}\|_{1, \infty} \\
& \leq \frac{\Delta t^{2}}{\epsilon_{0}} \frac{q}{m}\left\|\tilde{\boldsymbol{v}}^{n+1}\right\|_{m}^{2}(1+\sqrt{5})\|\nabla \overline{\boldsymbol{E}}\|_{1, \infty}
\end{aligned}
$$

where we have used 83 . This shows 82 and ends the proof. 


\section{Numerical experiments}

We now investigate the validity of the new ChECSIM algorithm, and compare its properties with the charge-conserving leap-frog (CCLF) and the ECSIM schemes, recalled in Sections 4.1 and 4.2 . These numerical experiments are performed with a $2 \mathrm{D}$ research code called Coffee which implements LagrangeNédélec finite elements on triangular meshes. We note that in a 2D setting the de Rham sequence is reduced. Corresponding to the choice of a weak Ampère equation, it reads

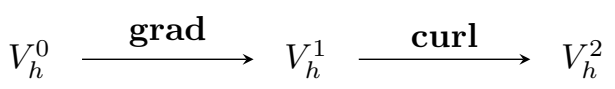

see, e.g. [14. Here,

$$
V_{h}^{2}=\mathbb{P}_{p-1}\left(\mathcal{T}_{h}\right):=\left\{u \in L^{2}(\Omega):\left.u\right|_{T} \in \mathbb{P}_{p-1}(T) \text { for } T \in \mathcal{T}_{h}\right\}
$$

corresponds to broken (discontinuous) piecewise polynomials of degree $p-1$,

$$
V_{h}^{1}=\left\{\boldsymbol{u} \in \boldsymbol{H}_{0}(\mathbf{c u r l}, \Omega):\left.u\right|_{T} \in \mathbb{P}_{p-1}(T)^{2}+\left(\begin{array}{c}
-y \\
x
\end{array}\right) \mathbb{P}_{p-1}(T) \text { for } T \in \mathcal{T}_{h}\right\}
$$

is the (first-kind) Nédélec finite element space of maximal degree $p$, and

$$
V_{h}^{0}=\mathcal{L}_{p}\left(\mathcal{T}_{h}\right):=\mathbb{P}_{p}\left(\mathcal{T}_{h}\right) \cap C_{0}(\Omega)
$$

is the Lagrange finite element space of degree $p \geq 1$. We refer e.g. to [6, 24, 20] for a complete description of these spaces.

Before describing our test cases, we may point out an interesting result from our simulations. As we have seen above, in the ChECSIM algorithm the velocity correction step 788 may not be feasible if the kinetic energy is lower than the field energy correction, and when that is not the case Proposition 5.4 guarantees that an adaptation of the time-step allows to have a well-posed correction, such as the one proposed in Appendix C. A first numerical observation is that for all the runs presented in this section, the correction step was always possible and we never had to adapt the time-step.

\subsection{Weibel instability}

In order to certify the validity of our alogrithms, we first simulate the electromagnetic Weibel instability [47. This phenomenon consists in the exponential growth of the self-induced magnetic field $B=B_{z}$ in a plasma where the velocity distribution of electrons is anisotropic, with higher kinetic energy in one of the physical dimensions. In our $2 \mathrm{D} 2 \mathrm{~V}$ simulation code, we reproduce the experiment documented in [18] and [29].

Here, we consider Equations (1)-(2) in a non-dimensional setting where the speed of light is set to $c=1$, as well as the electric permittivity of vacuum $\epsilon_{0}=1$, and the electron charge and mass $q_{e}=-1$ and $m_{e}=1$. 


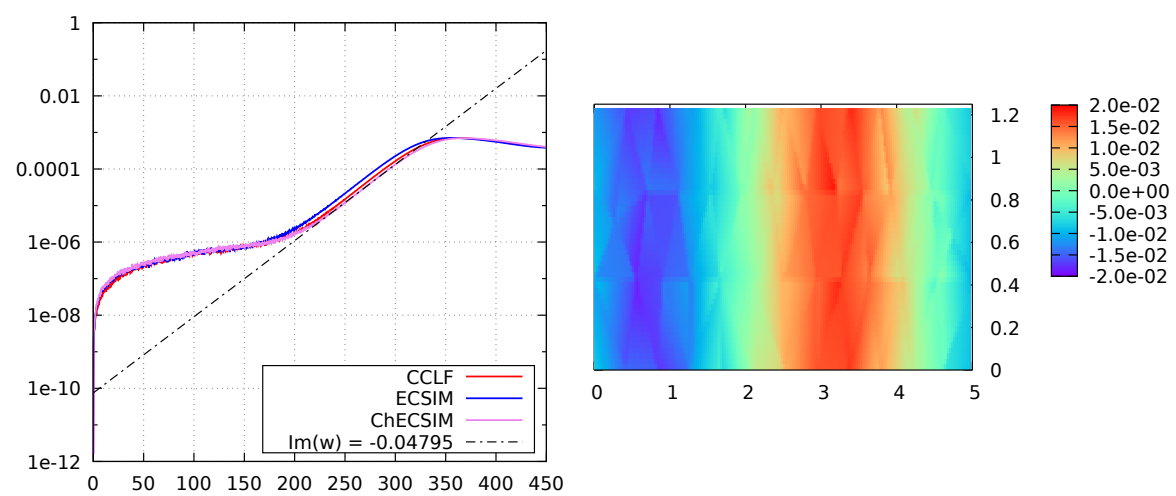

Figure 1: Weibel test-case : time evolution of the field energy using ChECSIM (left) and magnetic field B at $t=349.22 \omega_{p e}^{-1}$ (right).

The computational domain $\Omega$ is a periodic rectangle of length $L_{x}=2 \pi / k$, with $k=1.25$, and width $L_{y}=L_{x} / 4$. The domain is occupied by a plasma composed of electrons and a uniform neutralizing background of ions. The ions are considered to be fixed over time. At initial time, the electrons are distributed uniformly in space and have an anisotropic Maxwellian velocity distribution

$$
f^{0}(\boldsymbol{x}, \boldsymbol{v})=\frac{1}{2 \pi \sigma_{x} \sigma_{y}} \exp \left(\frac{1}{2}\left(\frac{v_{x}^{2}}{\sigma_{x}^{2}}+\frac{v_{y}^{2}}{\sigma_{y}^{2}}\right)\right)
$$

with $\sigma_{x}=\frac{0.02}{\sqrt{2}}$ and $\sigma_{y}=0.02 \sqrt{6}$, hence a higher thermal velocity in $y$. The initial magnetic field $B_{h}^{0} \in V_{h}^{2}$ is computed as an approximation of

$$
B^{0}(\boldsymbol{x})=\beta \cos (k x)
$$

$\{$ eq: weibel_b0 $\}$

with $\beta=-10^{-6}$, and the initial electric field $\boldsymbol{E}_{h}^{0} \in V_{h}^{1}$ is obtained from the initial particle distribution by solving the corresponding finite element Poisson problem and taking the gradient of the resulting discrete potential $\phi_{h}^{0} \in V_{h}^{0}$, in order to satisfy the discrete Gauss law (39) for $n=0$.

We recall that the Weibel instability corresponds to a magnetic mode of the form $B_{k}(t, \boldsymbol{x})=\widehat{B}_{k} e^{i(k x-\omega t)}$, with $\omega$ the most unstable solution of the dispersion relation [4].

$$
D(\omega, k):=\omega^{2}-k^{2}+\left(\frac{\sigma_{y}}{\sigma_{x}}\right)^{2}-1-\left(\frac{\sigma_{y}}{\sigma_{x}}\right)^{2} \Phi\left(\frac{\omega}{\sigma_{x} k}\right) \frac{\omega}{\sigma_{x} k}=0,
$$

i.e., the solution with largest positive imaginary part $\gamma:=\Im(\omega)>0$. Here $\Phi(z)=\exp \left(-1 / 2 z^{2}\right) \int_{-i \infty}^{z} \exp \left(1 / 2 \xi^{2}\right) d \xi$, and the analytic growth rate of the 
instability is $\gamma \approx 0.02784$, see eg [29]. We shall verify whether this rate is obtained in the numerical simulations.

We simulate the plasma during a time $T=450$. The domain is discretized by a conforming triangular mesh $\mathcal{T}_{h}$ composed of 74 elements of typical diameter $h=0.5585$. The order of the finite element spaces (84) is $p=2$, and the number of numerical macro-particles used in the PIC approximation is $N=2.96 \cdot 10^{5}$. The time-step used is $\Delta t=0.05$, corresponding to approximately $80 \%$ of the maximum stable time-step for the explicit leap-frog scheme for the resulting Finite Element Maxwell solver.

On Figure 1, we present the time evolution of the magnetic energy $\frac{1}{2}\left\|B_{h}^{n}\right\|^{2}$, computed with each of the three schemes presented in this article. Then, we present on Figure 2 the time evolution of the relative errors on the conservation of the total discrete energy (42),

$$
\frac{\left|\mathcal{E}_{h}\left(\boldsymbol{V}^{n}, \boldsymbol{e}^{n}, \boldsymbol{b}^{n}\right)-\mathcal{E}_{h}\left(\boldsymbol{V}^{0}, \boldsymbol{e}^{0}, \boldsymbol{b}^{0}\right)\right|}{\left|\mathcal{E}_{h}\left(\boldsymbol{V}^{0}, \boldsymbol{e}^{0}, \boldsymbol{b}^{0}\right)\right|}
$$

and on the electric Gauss law (39),

$$
\frac{\left\|\operatorname{div}_{h} \boldsymbol{E}_{h}^{n}-\rho_{h}^{n}\right\|}{\max \left(\left\|\operatorname{div}_{h} \boldsymbol{E}_{h}^{n}\right\|,\left\|\rho_{h}^{n}\right\|\right)} .
$$

On Figure 1, the exponential growth of the magnetic energy is clearly visible, and it is comparable for the three schemes. Since this energy depends on the square of the norm of $B$, its growth rate is twice that of the field. A linear fit of the ChECSIM curve between $t=250$ and 310 yields a growth rate of $\gamma \approx 0.024$, in good agreement with the analytical value.

On the left of figure 2, we observe that the relative energy conservation (91) is close to machine accuracy for ECSIM and ChECSIM (a slight increase is visible for ChECSIM, but still on the order of roundoff errors). With CCLF the energy
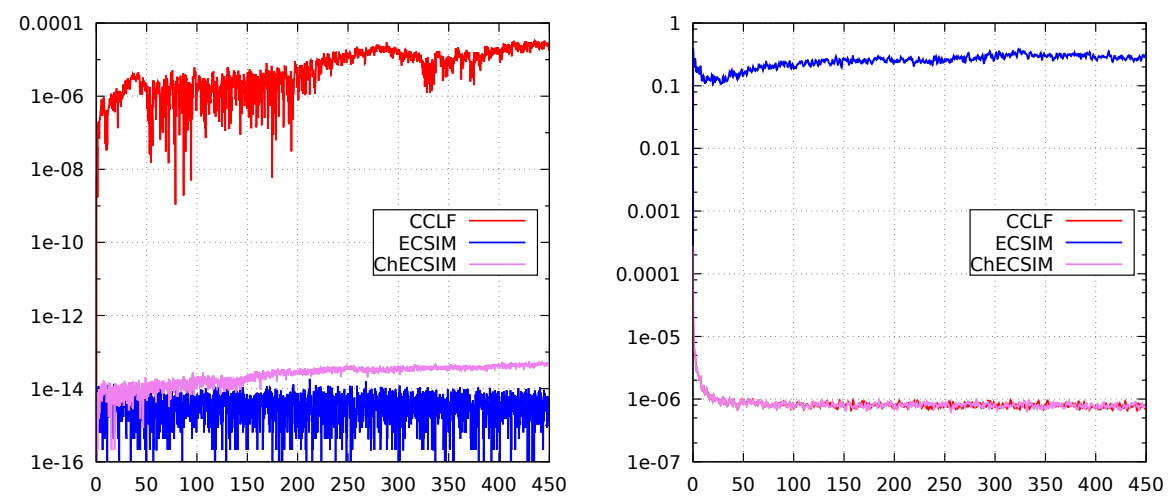

Figure 2: Weibel test-case : time evolution of the quantities 91 and 92 corresponding to the relative errors on the energy conservation (left) and on electric Gauss's law (right). 
is also rather stable, but the conservation error is higher by about 6 orders of magnitude. On the right of the same figure, we observe that both ChECSIM and CCLF preserve Gauss's law with high accuracy. Note that although this law should be exactly satisfied by these two schemes, we observe relative errors which are bigger than round-off : this is due to approximations when tracking the particles through the domain, and could be reduced with further work on the implementation. For ECSIM, we observe that the error on the electric Gauss law is significantly larger, again by about 6 orders of magnitude.

This provides us with a first numerical validation of the basic properties of the ChECSIM scheme, namely the conservation of energy and charge for an isolated system.

\subsection{Electron beam in a simple diode}

To assess the properties of the different schemes in a more realistic setting where the system is no longer isolated, we now consider a second test-case consisting of an electron beam in a simple, academic diode.

Following [42, we consider a diode modeled by a square domain $\Omega$ of dimensions $L_{x}=L_{y}=0.1 \mathrm{~m}$. In this domain, electrons are emitted at a cathode lying in the face of abscissa $x=0$, and accelerated towards the anode, lying in the face of abscissa $x=0.1$, by a constant exterior electric field $\boldsymbol{E}_{\text {ext }}=-\left(U_{\text {ext }} / L_{x}\right) \hat{\boldsymbol{x}}$ with an operating voltage $U_{\text {ext }}=10^{5} \mathrm{~V}$ (see Appendix B for the modeling of exterior fields in the ECSIM and ChECSIM schemes). More precisely, the electrons are injected in the domain through a portion of the cathode : a strip centered at $y=0.05$ and of fixed width $L_{\text {inj }}=0.03786 \mathrm{~m}$. The current density at the emission surface is $I=-5536 \mathrm{~A} . \mathrm{m}^{-2}$, corresponding to $75 \%$ of the space-charge limiting current density.

The electron beam is approximated by numerical macro-particles emitted at rate $r=9000 \mathrm{~ns}^{-1}$. In this $2 \mathrm{D}$ model, each numerical particle carries a linear density $w$ of electrons in the $z$ dimension. Thus, the the total charge injected during a time $\Delta t$ reads $Q_{\mathrm{inj}}(\Delta t):=I L_{z} L_{\mathrm{inj}} \Delta t=r \Delta t w q_{e} L_{z}$ where $L_{z}$ is the height of the $3 \mathrm{D}$ diode, $q_{e}$ is the charge of an electron and $w$ is the uniform weight of the $2 \mathrm{D}$ macro-particles, which has the physical dimension of a linear density. We then obtain $w \approx 1.45 \cdot 10^{8} \mathrm{~m}^{-1}$.

The domain is discretized by an unstructured conforming triangular mesh $\mathcal{T}_{h}$, composed of 2158 elements, the typical diameter of a cell being $h=0.005 \mathrm{~m}$. The electromagnetic fields are approached by the structure-preserving finite element complex (84), with a precision order $p=2$.

We simulate the flow of electrons within the diode during a time of $T=50 \mathrm{~ns}$, using the three schemes presented in this article : the charge-conserving leapfrog scheme (CCLF), ECSIM and ChECSIM. We note that for this test case the energy is not expected to remain constant, as the system is not isolated. Up to $T_{S}=3 \mathrm{~ns}$ approximately, the system is in a transitory regime : the electronic current sets up in the initially empty diode. Then, from $T_{S}$ to $T$, the phase-space density of particles and the electromagnetic fields are approximately constant, and the system is in a stationary regime. These two phases are clearly visible on 


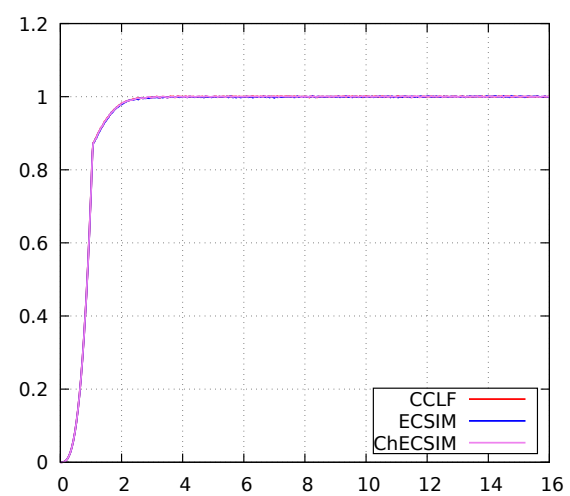

(a) $\Delta t_{\text {stat }}=\Delta t_{\mathrm{CFL}}$

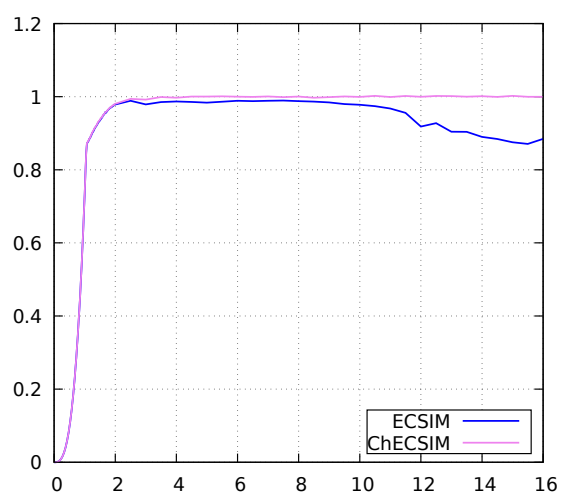

(b) $\Delta t_{\text {stat }}=10 \Delta t_{\mathrm{CFL}}$

Figure 3: Diode test-case : time evolution of the total energy 42. Here, $t$ is expressed in nanoseconds while the energy is normalized.

the left panel of Figure 3, where we plot the time evolution of the total energy (42) in the diode.

Specifically, Figure 3 displays two sets of energy curves: on the left panel the runs are obtained using a constant time-step $\Delta t_{\mathrm{CFL}}=1.25 \cdot 10^{-3} \mathrm{~ns}$, corresponding to $80 \%$ of the CFL time-step of the leap-frog Maxwell solver. This constraint is needed for the stability of the CCLF scheme. On the right panel, the curves correspond to ECSIM and ChECSIM runs using a larger time step. Indeed these methods are not constrained by an explicit CFL condition, so it is interesting to compare their results when the time step is increased. In order to have a similar accuracy for the transitory regime, we have kept the time-step $\Delta t_{\mathrm{CFL}}$ up to time $T_{S}$, and then switched to a longer $\Delta t_{\text {stat }}$ for the stationary phase.

Then on Figures 4 and 5 we plot the profiles of the numerical electron beam and of the $E_{x}$ field at the final time $T$. Again, several values are used for the time-step $\Delta t_{\text {stat }}$, as described above.

On the left of Figure 3, we observe a stationary total energy from $t=T_{S}$ to $T$ with all three schemes. On the right, one notices that for the longest choosen time-step $\Delta t_{\mathrm{stat}}=10 \Delta t_{\mathrm{CFL}}$, a significant fraction of the energy is lost by ECSIM. Although this may seem surprising, we emphasize that it does not contradict the theoretical properties of the energy conserving scheme, since they are only guaranteed for an isolated system. Here we are not in this situation : particles enter and leave the domain, they gain energy from the external field, and absorbing boundary conditions on the horizontal boundaries allow some of the field energy to be dissipated. For the intermediate time-step $\Delta t_{\text {stat }}=$ $2 \Delta t_{\mathrm{CFL}}$ (not shown here) the energy profile is similar for ECSIM and ChECSIM, but we have observed that the total energy also decreases significantly with ECSIM in longer simulations with $T=50$ ns. For ChECSIM we observe that the energy is well preserved during the stationary phase, even when longer 
time-steps are used. We should point out that this behaviour is not a direct consequence of the sole energy conservation properties of the scheme since the system is not isolated, but rather an indication of the improved stability of the ChECSIM algorithm.

We finally turn to the profiles of the particle beam and the electric field on Figures 4 and 5 . For the smallest time-step $\Delta t_{\text {stat }}=\Delta t_{\mathrm{CFL}}$, the CCLF profiles on panels $4 \mathrm{a}$ and $5 \mathrm{a}$ show a good qualitative behavior, very similar to those obtained with ChECSIM (not shown here). Here we note that the moiré patterns on the beams are due to the pseudo-random sampling of particles in the injection zone. The profiles for the ECSIM run in panels $4 \mathrm{~b}$ and $5 \mathrm{~b}$ are close, despite some subtle filamentation developping in the upper half of the particle beam (a typical result of small space-charge errors due to the lack of charge-conservation, see e.g. (4), and a slightly stronger electric field at the cathode.

For the time-step $\Delta t_{\text {stat }}=2 \Delta t_{\mathrm{CFL}}$, the ChECSIM profiles in panels $4 \mathrm{c}$ and $5 \mathrm{c}$ are virtually identical, but in the ECSIM run the numerical artifacts grow stronger: the filamentation of the beam is more pronounced in panel 4d, and the corresponding field in panel $5 \mathrm{~d}$ is now higher at the cathode by around $15 \%$. With the longest time-step $\Delta t_{\text {stat }}=10 \Delta t_{\text {CFL }}$, the profiles obtained with ChECSIM on panels $4 \mathrm{e}$ and $5 \mathrm{e}$ remain very close to the previous ones, despite a higher level of noise visible in the field. This is no longer the case with the ECSIM run, where the accumulation of charge errors leads to significant errors: on panel $4 \mathrm{f}$ we see that the particle beam is badly scattered, and on panel $5 \mathrm{f}$ the field takes clearly unphysical values at the cathode, and is also disrupted by strong spurious oscillations close to the anode.

As a result, we find that this test-case completes the numerical evidence of Section 6.1 and confirms that the combined energy and charge-conserving properties of the ChECSIM algorithm lead to an improved numerical stability for long-time simulations.

\section{Conclusion}

In this article, we have reformulated the ECSIM algorithm in a structurepreserving finite element setting and we have used the properties of this general discrete framework to complement the original energy-conserving method with an explicit charge-conserving correction step based on FEM-PIC conservative current deposition techniques. The resulting ChECSIM scheme is still linearly implicit in the fields and explicit in the particles, and it now preserves exactly the total energy and the discrete charge in a sense that is compatible with the structure-preserving finite element framework. A condition for our correction step to be well-defined is that the kinetic energy is higher than the field correction energy, and we have provided a rigorous criterion on the time step, under which this condition is always fulfilled. In practice one may need to dynamically adapt the time-step to meet this criterion, but this was not the case in the numerical experiments performed in this article. These experiments have allowed us to exhibit a clear improvement of the stability properties of the new scheme, 


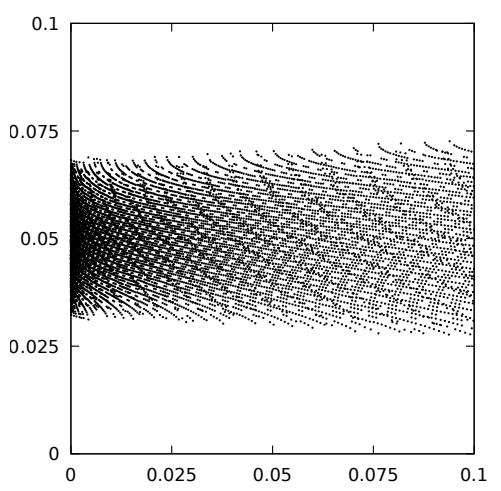

(a) CCLF with $\Delta t_{\text {stat }}=\Delta t_{\mathrm{CFL}}$

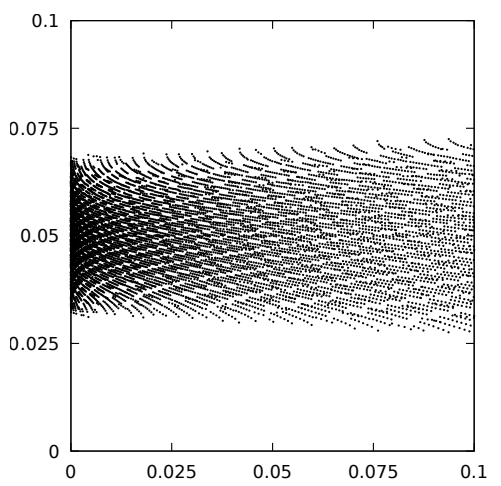

(c) ChECSIM with $\Delta t_{\text {stat }}=2 \Delta t_{\mathrm{CFL}}$

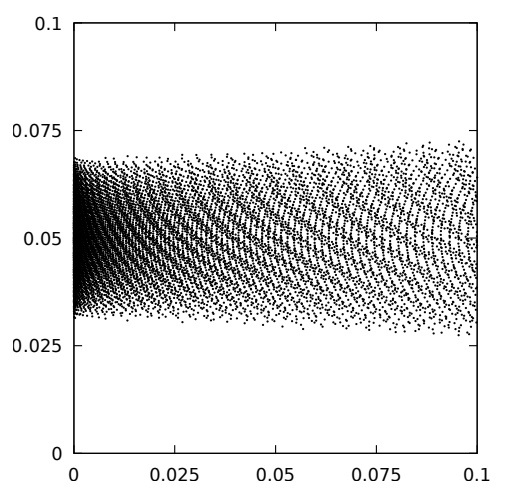

(e) ChECSIM with $\Delta t_{\text {stat }}=10 \Delta t_{\text {CFL }}$

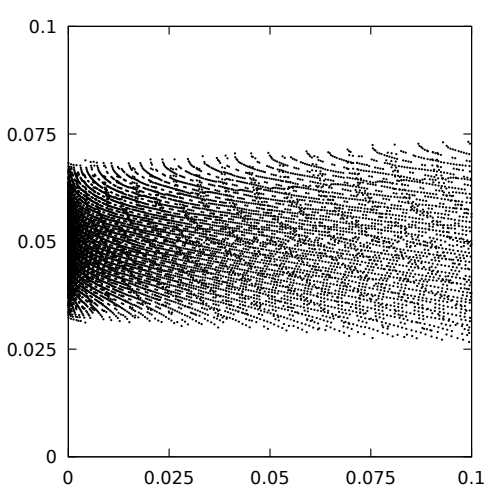

(b) ECSIM with $\Delta t_{\text {stat }}=\Delta t_{\mathrm{CFL}}$

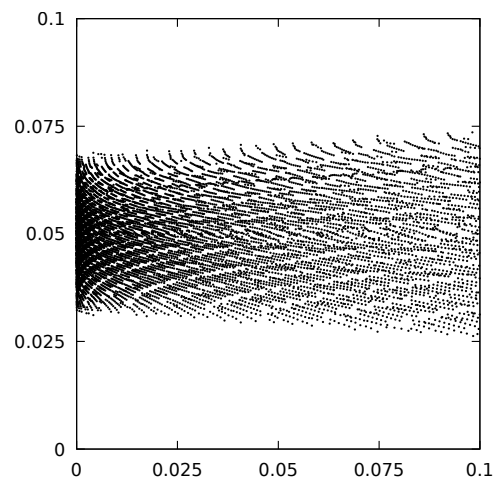

(d) ECSIM with $\Delta t_{\text {stat }}=2 \Delta t_{\text {CFL }}$

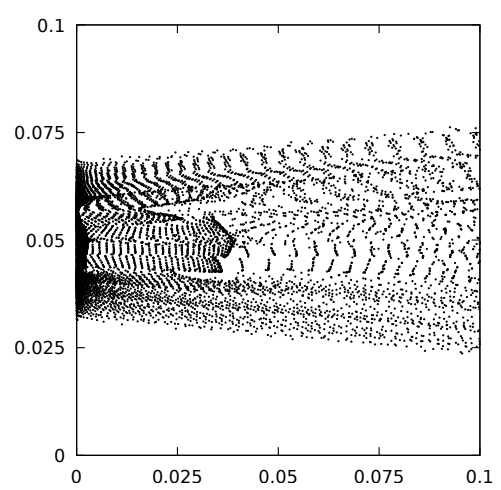

(f) ECSIM with $\Delta t_{\text {stat }}=10 \Delta t_{\mathrm{CFL}}$

Figure 4: Diode test-case: positions of the numerical particles showing the beam profile at $t=16 \mathrm{~ns}$ for various schemes and time-steps. The values on the $x$ and $y$ axes are in meters. 


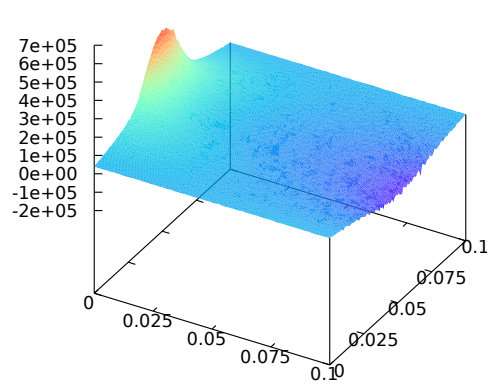

(a) CCLF with $\Delta t_{\text {stat }}=\Delta t_{\mathrm{CFL}}$

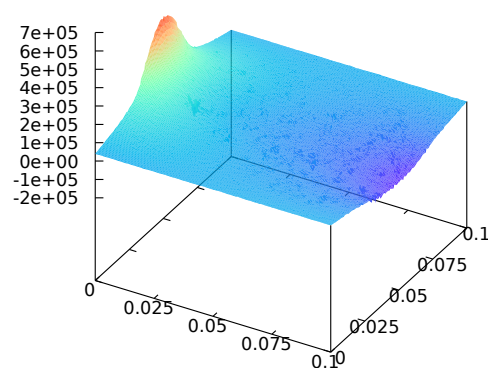

(c) ChECSIM with $\Delta t_{\text {stat }}=2 \Delta t_{\mathrm{CFL}}$

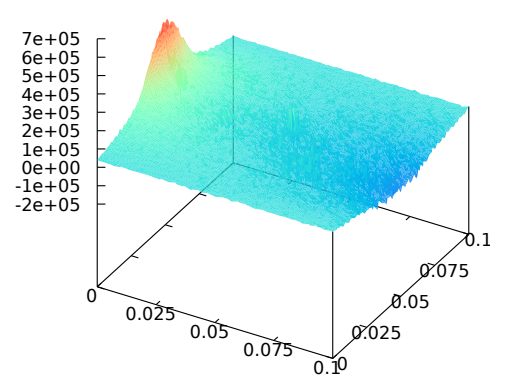

(e) ChECSIM with $\Delta t_{\text {stat }}=10 \Delta t_{\mathrm{CFL}}$

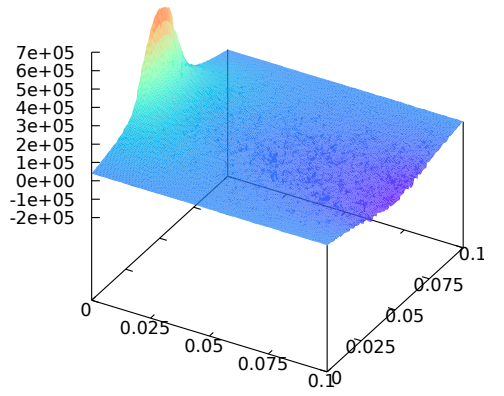

(b) ECSIM with $\Delta t_{\text {stat }}=\Delta t_{\mathrm{CFL}}$

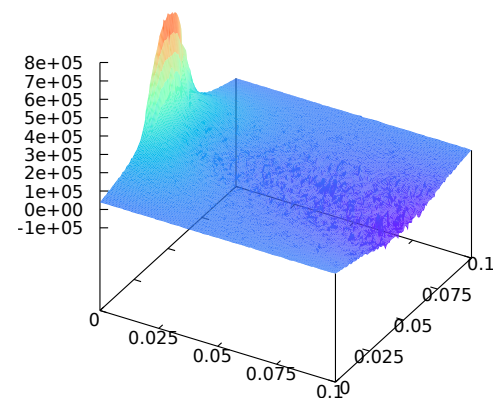

(d) ECSIM with $\Delta t_{\text {stat }}=2 \Delta t_{\mathrm{CFL}}$

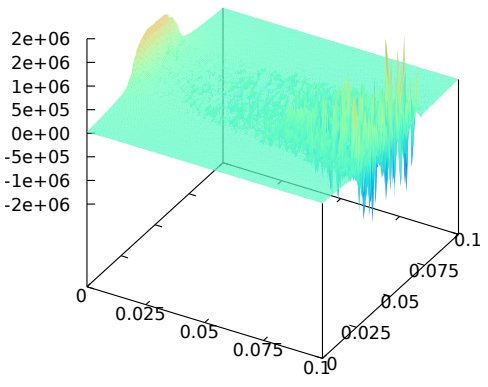

(f) ECSIM with $\Delta t_{\text {stat }}=10 \Delta t_{\mathrm{CFL}}$

Figure 5: Diode test-case: profile of the $E_{x}$ field at $t=16 \mathrm{~ns}$ for various schemes and timesteps. The values on the $x$ and $y$ axes are in meters, those on the $z$ axis are in volt/meter. 
in particular for configurations involving large time steps and a modeled system that is not isolated from exterior energy sources.

\section{Acknowledgements}

The authors would like to thank Benedikt Perse, Katharina Kormann and Eric Sonnendrücker for fruitful discussions.

\section{Appendix A. Modelling of absorbing boundary conditions}

Absorbing boundaries may be modelled by decomposing the boundary $\Gamma:=$ $\partial \Omega$ into a part $\Gamma_{M}$ with metallic boundary conditions and an part $\Gamma_{A}$ with absorbing Silver-Müller conditions [3], so that (13) is replaced by

$$
\boldsymbol{n} \times \boldsymbol{E}= \begin{cases}0 & \text { on } \Gamma_{M} \\ -\boldsymbol{n} \times(\boldsymbol{n} \times c \boldsymbol{B}) & \text { on } \Gamma_{A} .\end{cases}
$$

Using this boundary condition in the integration by parts, the weak Ampère equation 15 becomes

$$
\left\langle\partial_{t} \boldsymbol{E}(t), \boldsymbol{\phi}^{1}\right\rangle-\left\langle\boldsymbol{B}(t), \operatorname{curl} \boldsymbol{\phi}^{1}\right\rangle+c\left\langle\boldsymbol{n} \times \boldsymbol{E}, \boldsymbol{n} \times \boldsymbol{\phi}^{1}\right\rangle_{\Gamma_{A}}=-\left\langle\boldsymbol{J}(t), \boldsymbol{\phi}^{1}\right\rangle
$$

$\{$ eq:var-ampere-abc $\}$

for all $\phi^{1} \in \mathcal{V}^{1}$, with an electric field now sought for in the space $C^{1}\left([0, T] ; \mathcal{V}^{1}\right)$ with

$$
\mathcal{V}^{1}=\boldsymbol{H}_{0, \Gamma_{M}}(\operatorname{curl}, \Omega)=\left\{\boldsymbol{u} \in \boldsymbol{H}(\operatorname{curl}, \Omega): \boldsymbol{n} \times \boldsymbol{u}=0 \text { on } \Gamma_{M}\right\} .
$$

Accordingly, the finite element space $\mathcal{V}_{h}^{1}$ should have non-homogeneous boundary conditions on $\Gamma_{A}$, and the time-continous matrix Ampère equation (28) becomes

$$
\mathbb{M}_{1} \frac{\mathrm{d}}{\mathrm{d} t} \boldsymbol{e}-\left(\mathbb{M}_{2} \mathbb{C}\right)^{T} \boldsymbol{b}+c \mathbb{T}_{\Gamma_{A}} \boldsymbol{e}=-\boldsymbol{j}
$$

$\{$ Ah-mat-abc $\}$

where $\mathbb{T}_{A}$ is a boundary mass-matrix involving the tangential traces on the absorbing boundary $\Gamma_{A}$,

$$
\mathbb{T}_{\Gamma_{A}}=\left(\left\langle\boldsymbol{n} \times \Lambda_{i}^{1}, \boldsymbol{n} \times \Lambda_{j}^{1}\right\rangle_{\Gamma_{A}}\right)_{1 \leq i, j \leq N_{1}} .
$$

Note that if $\Gamma_{A}=\emptyset$, then $\mathbb{T}_{\Gamma_{A}}=0$ and we recover Equation 28. Using an semi-implicit time-stepping for the absorbing boundary terms, the Maxwell solver in the leap-frog scheme then becomes

$$
\left\{\begin{aligned}
\boldsymbol{b}^{n+\frac{1}{2}}-\boldsymbol{b}^{n} & =-\frac{\Delta t}{2} \mathbb{C} e^{n} \\
\mathbb{M}_{1}\left(\boldsymbol{e}^{n+1}-\boldsymbol{e}^{n}\right) & =\Delta t\left(c^{2}\left(\mathbb{M}_{2} \mathbb{C}\right)^{T} \boldsymbol{b}^{n+\frac{1}{2}}-c \mathbb{T}_{\Gamma_{A}}\left(\frac{\boldsymbol{e}^{n+1}+\boldsymbol{e}^{n}}{2}\right)-\frac{1}{\epsilon_{0}} \boldsymbol{j}^{n+\frac{1}{2}}\right) \\
\boldsymbol{b}^{n+1}-\boldsymbol{b}^{n+\frac{1}{2}} & =-\frac{\Delta t}{2} \mathbb{C} \boldsymbol{e}^{n+1}
\end{aligned}\right.
$$


Similarly, the fully implicit Maxwell solver (44) in the ECSIM scheme takes the form

$$
\left\{\begin{aligned}
\mathbb{M}_{1}\left(\boldsymbol{e}^{n+1}-\boldsymbol{e}^{n}\right) & =\Delta t\left(c^{2}\left(\mathbb{M}_{2} \mathbb{C}\right)^{T} \boldsymbol{b}^{n+\frac{1}{2}}-c \mathbb{T}_{\Gamma_{A}} e^{n+\frac{1}{2}}-\frac{1}{\epsilon_{0}} j^{n+\frac{1}{2}}\right) \\
\boldsymbol{b}^{n+1}-\boldsymbol{b}^{n} & =-\Delta t \mathbb{C} e^{n+\frac{1}{2}}
\end{aligned}\right.
$$

and the corresponding linearly implicit formulation $(50)$ becomes

$$
\begin{aligned}
\left(\begin{array}{cc}
\mathbb{M}_{1}+\frac{c \Delta t}{2} \mathbb{T}_{\Gamma_{A}}+\frac{\Delta t^{2}}{4 \epsilon_{0}} \mathbb{L}^{n} & -\frac{c^{2} \Delta t}{2}\left(\mathbb{M}_{2} \mathbb{C}\right)^{T} \\
\frac{\Delta t}{2} \mathbb{C} & \mathbb{I}
\end{array}\right)\left(\begin{array}{l}
\boldsymbol{e}^{n+1} \\
\boldsymbol{b}^{n+1}
\end{array}\right) \\
=\left(\begin{array}{cc}
\mathbb{M}_{1}-\frac{c \Delta t}{2} \mathbb{T}_{\Gamma_{A}}-\frac{\Delta t^{2}}{4 \epsilon_{0}} \mathbb{L}^{n} & \frac{c^{2} \Delta t}{2}\left(\mathbb{M}_{2} \mathbb{C}\right)^{T} \\
-\frac{\Delta t}{2} \mathbb{C} & \mathbb{I}
\end{array}\right)\left(\begin{array}{c}
\boldsymbol{e}^{n} \\
\boldsymbol{b}^{n}
\end{array}\right)-\frac{\Delta t}{\epsilon_{0}}\left(\begin{array}{c}
\boldsymbol{j}_{\exp }^{n} \\
0
\end{array}\right)
\end{aligned}
$$

The same applies for the ChECSIM guess step, where the linearly implicit formulation 72 becomes

$$
\begin{aligned}
& \left(\begin{array}{cc}
\mathbb{M}_{1}+\frac{c \Delta t}{2} \mathbb{T}_{\Gamma_{A}}+\frac{\Delta t^{2}}{4 \epsilon_{0}} \overline{\mathbb{L}}^{n} & -\frac{c^{2} \Delta t}{2}\left(\mathbb{M}_{2} \mathbb{C}\right)^{T} \\
\frac{\Delta t}{2} \mathbb{C} & \mathbb{I}
\end{array}\right)\left(\begin{array}{l}
\tilde{\boldsymbol{e}}^{n+1} \\
\boldsymbol{b}^{n+1}
\end{array}\right) \\
& =\left(\begin{array}{cc}
\mathbb{M}_{1}-\frac{c \Delta t}{2} \mathbb{T}_{\Gamma_{A}}-\frac{\Delta t^{2}}{4 \epsilon_{0}} \overline{\mathbb{L}}^{n} & \frac{c^{2} \Delta t}{2}\left(\mathbb{M}_{2} \mathbb{C}\right)^{T} \\
-\frac{\Delta t}{2} \mathbb{C} & \mathbb{I}
\end{array}\right)\left(\begin{array}{c}
\boldsymbol{e}^{n} \\
\boldsymbol{b}^{n}
\end{array}\right)-\frac{\Delta t}{\epsilon_{0}}\left(\begin{array}{c}
\tilde{\boldsymbol{\jmath}}_{\exp }^{n} \\
0
\end{array}\right) .
\end{aligned}
$$

Finally in order to obtain an electric field $e^{n+1}$ satisfying the Ampère matrix equation

$$
\mathbb{M}_{1}\left(\boldsymbol{e}^{n+1}-\boldsymbol{e}^{n}\right)=\Delta t\left(c^{2}\left(\mathbb{M}_{2} \mathbb{C}\right)^{T} \boldsymbol{b}^{n+\frac{1}{2}}-c \mathbb{T}_{\Gamma_{A}} \boldsymbol{e}^{n+\frac{1}{2}}-\frac{1}{\epsilon_{0}} \boldsymbol{j}^{n+\frac{1}{2}}\right)
$$

with the charge-conserving current term $\boldsymbol{j}^{n+\frac{1}{2}}=\frac{1}{2}\left(\boldsymbol{j}^{n,-}+\boldsymbol{j}^{n,+}\right)$, the field correction step (78) rewrites as

$$
\left(\mathbb{M}_{1}+\frac{c \Delta t}{2} \mathbb{T}_{\Gamma_{A}}\right)\left(\boldsymbol{e}^{n+1}-\tilde{\boldsymbol{e}}^{n+1}\right)=-\frac{\Delta t}{2 \epsilon_{0}}\left(\boldsymbol{j}^{n,+}-\tilde{\boldsymbol{j}}^{n,-}\right) .
$$

$\{$ corr-checsim-abc

\section{Appendix B. Influence of applied exterior fields}

In the case where an exterior field $\left(\boldsymbol{E}_{\text {ext }}, \boldsymbol{B}_{\text {ext }}\right)$ is applied, we point out that the linearly-implicit formulation (50) of the ECSIM scheme needs to be modified in a non-trivial way. Indeed, the velocity kick in 43 becomes

$$
\boldsymbol{v}_{k}^{n+\frac{1}{2}}=\frac{1}{2}\left(\boldsymbol{v}_{k}^{n}+\boldsymbol{v}_{k}^{n+1}\right)=\boldsymbol{v}_{k}^{n}+\frac{q \Delta t}{2 m}\left(\left(\boldsymbol{E}_{h}^{n+\frac{1}{2}}+\boldsymbol{E}_{\text {ext }}\right)\left(\boldsymbol{x}_{k}^{n+\frac{1}{2}}\right)+\widehat{\mathbb{B}}_{k}^{n} \boldsymbol{v}_{k}^{n+\frac{1}{2}}\right)
$$

with a matrix $\widehat{\mathbb{B}}_{k}^{n}$ such that $\widehat{\mathbb{B}}_{k}^{n} \boldsymbol{v}=\boldsymbol{v} \times\left(\boldsymbol{B}_{h}^{n}+\boldsymbol{B}_{\text {ext }}\right)\left(\boldsymbol{x}_{k}^{n+\frac{1}{2}}\right)$. Therefore we need to modify the matrix $\mathbb{A}_{k}^{n}$ accordingly,

$$
\widehat{\mathbb{A}}_{k}^{n}:=\left(\mathbb{I}-\eta_{k} \widehat{\mathbb{B}}_{k}^{n}\right)^{-1}
$$


and to decompose the fully implicit current 45 into

$$
\boldsymbol{j}^{n+\frac{1}{2}}=\boldsymbol{j}_{\text {exp }}^{n}+\boldsymbol{j}_{\text {imp }}^{n}\left[\boldsymbol{E}_{h}^{n+\frac{1}{2}}+\boldsymbol{E}_{\text {ext }}\right]
$$

with an explicit part $\boldsymbol{j}_{\exp }^{n}$ defined similarly as in 48), and an implicit part which now involves the exterior field. Namely, we have

$$
\left\{\begin{aligned}
& \boldsymbol{j}_{\mathrm{exp}}^{n}=\left(\sum_{k} q_{k} \widehat{\mathbb{A}}_{k}^{n} \boldsymbol{v}_{k}^{n} \cdot \Lambda_{i}^{1}\left(\boldsymbol{x}^{n+\frac{1}{2}}\right)\right)_{1 \leq i \leq N_{1}}, \\
& \boldsymbol{j}_{\mathrm{imp}}^{n}\left[\boldsymbol{E}_{h}^{n+\frac{1}{2}}+\boldsymbol{E}_{\mathrm{ext}}\right]=\frac{\Delta t}{2}\left(\sum_{k} \frac{q_{k}^{2}}{m_{k}}\left(\widehat{\mathbb{A}}_{k}^{n}\left(\boldsymbol{E}_{h}^{n+\frac{1}{2}}+\boldsymbol{E}_{\mathrm{ext}}\right)\left(\boldsymbol{x}_{k}^{n+\frac{1}{2}}\right)\right) \cdot \Lambda_{i}^{1}\left(\boldsymbol{x}^{n+\frac{1}{2}}\right)\right)_{1 \leq i \leq N_{1}} \\
&=\frac{\Delta t}{2} \widehat{\mathbb{L}}^{n}\left(\frac{\boldsymbol{e}^{n}+\boldsymbol{e}^{n+1}}{2}+\boldsymbol{e}_{\mathrm{ext}}\right) .
\end{aligned}\right.
$$

where the matrix $\widehat{\mathbb{L}}^{n}$ is defined as in $(49)$, with the proper correction on the

magnetic matrices. In particular, the linearly-implicit Maxwell system 50 becomes

$$
\begin{aligned}
& \left(\begin{array}{cc}
\mathbb{M}_{1}+\frac{\Delta t^{2}}{4 \epsilon_{0}} \widehat{\mathbb{L}}^{n} & -\frac{c^{2} \Delta t}{2}\left(\mathbb{M}_{2} \mathbb{C}\right)^{T} \\
\frac{\Delta t}{2} \mathbb{C} & \mathbb{I}
\end{array}\right)\left(\begin{array}{l}
e^{n+1} \\
\boldsymbol{b}^{n+1}
\end{array}\right) \\
& \quad=\left(\begin{array}{cc}
\mathbb{M}_{1}-\frac{\Delta t^{2}}{4 \epsilon_{0}} \widehat{\mathbb{L}}^{n} & \frac{c^{2} \Delta t}{2}\left(\mathbb{M}_{2} \mathbb{C}\right)^{T} \\
-\frac{\Delta t}{2} \mathbb{C} & \mathbb{I}
\end{array}\right)\left(\begin{array}{l}
\boldsymbol{e}^{n} \\
\boldsymbol{b}^{n}
\end{array}\right)-\frac{\Delta t}{\epsilon_{0}}\left(\begin{array}{c}
\boldsymbol{j}_{\exp }^{n}+\frac{\Delta t}{2} \widehat{\mathbb{L}}^{n} \boldsymbol{e}_{\text {ext }} \\
0
\end{array}\right)
\end{aligned}
$$

$\{$ Max-li-ext $\}$

where we see that the exterior electric field also appears as an additional source term. In the ChECSIM guess step, the modification is exactly the same.

\section{Appendix C. Dynamic adaptation of the time-step}

In the ChECSIM algorithm, the velocity correction step 788 is only welldefined when the kinetic energy is larger than the variation of the field energy due to the charge-conserving current correction. Although this was always the case in the numerical experiments conducted for this article, it may no longer be true when the particles have a low velocity or when the field correction is important. Since Proposition 5.4 guarantees that the correction step is wellposed for a time-step small enough, in practice one may use a dynamic recursive splitting of the time-step, such as the following one. 


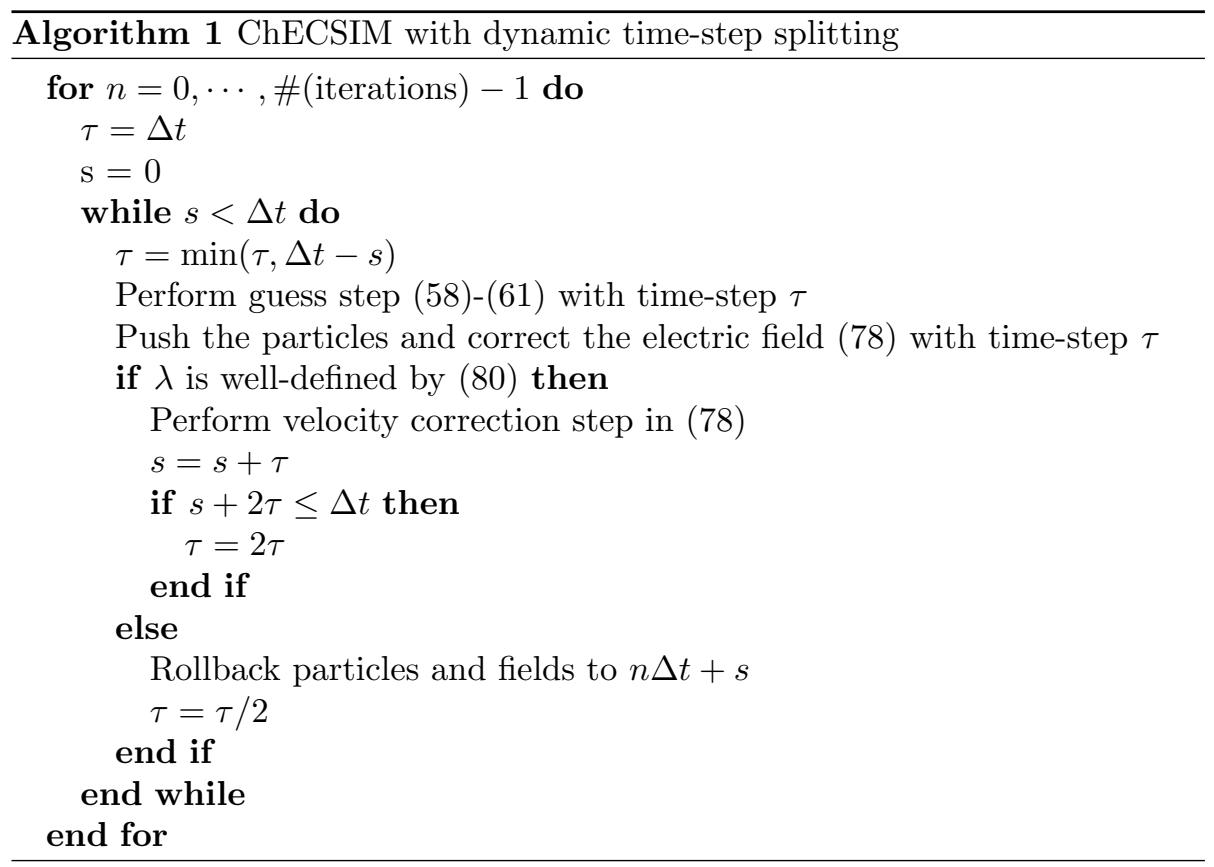

[1] Douglas N. Arnold, Richard S. Falk, and Ragnar Winther. Finite element exterior calculus, homological techniques, and applications. Acta Numer., $15: 1-155,2006$.

[2] Douglas N. Arnold, Richard S. Falk, and Ragnar Winther. Finite element exterior calculus: from Hodge theory to numerical stability. Bull. Amer. Math. Soc. (N.S.), 47(2):281-354, 2010.

[3] Franck Assous, Patrick Ciarlet, and Simon Labrunie. Mathematical foundations of computational electromagnetism. Applied Mathematical Sciences, Vol 198. Springer, 2018.

[4] R Barthelmé and C Parzani. Numerical charge conservation in particle-incell codes. In Numerical methods for hyperbolic and kinetic problems, pages 7-28. Eur. Math. Soc., Zürich, 2005.

[5] C K Birdsall and A.B. Langdon. Plasma physics via computer simulation. Adam Hilger, IOP Publishing, 1991.

[6] Daniele Boffi, Franco Brezzi, and Michel Fortin. Mixed finite element methods and applications, volume 44 of Springer Series in Computational Mathematics. Springer, 2013.

[7] J.P. Boris. Relativistic plasma simulation-optimization of a hybrid code. In Fourth Conf Num Sim Plasmas, 1970. Proc. Fourth Conf. Num. Sim. Plasmas, 1971. 
[8] Alain Bossavit. Computational electromagnetism: variational formulations, complementarity, edge elements. Academic Press, 1998.

[9] A. Buffa, G. Sangalli, and R. Vázquez. Isogeometric analysis in electromagnetics: B-splines approximation. Computer Methods in Applied Mechanics and Engineering, 199(17):1143-1152, March 2010.

[10] Annalisa Buffa, J Rivas, G Sangalli, and R Vázquez. Isogeometric discrete differential forms in three dimensions. SIAM Journal on Numerical Analysis, 49(2):818-844, 2011.

[11] Martin Campos Pinto, Sébastien Jund, Stéphanie Salmon, and Éric Sonnendrücker. Charge-conserving FEM-PIC schemes on general grids. Comptes Rendus Mécanique, 342(10):570-582, October 2014.

[12] Martin Campos Pinto and Eric Sonnendrücker. Gauss-compatible Galerkin schemes for time-dependent Maxwell equations. Math. Comp., 85(302):2651-2685, 2016.

[13] Martin Campos Pinto and Eric Sonnendrücker. Compatible Maxwell solvers with particles I: conforming and non-conforming $2 \mathrm{~d}$ schemes with a strong Ampere law. The SMAI journal of computational mathematics, 3:53-89, 2017.

[14] Martin Campos Pinto and Eric Sonnendrücker. Compatible Maxwell solvers with particles II: conforming and non-conforming $2 \mathrm{~d}$ schemes with a strong Faraday law. The SMAI journal of computational mathematics, 3:91-116, 2017.

[15] L. Chacón, G. Chen, and D. C. Barnes. A charge- and energy-conserving implicit, electrostatic particle-in-cell algorithm on mapped computational meshes. Journal of Computational Physics, 233:1-9, January 2013.

[16] Yuxi Chen and Gábor Tóth. Gauss's Law satisfying Energy-Conserving Semi-Implicit Particle-in-Cell method. Journal of Computational Physics, 386:632-652, June 2019.

[17] S.J. Cooke, K.T. Nguyen, A.N. Vlasov, T.M. Antonsen, B. Levush, T.A. Hargreaves, and M.F. Kirshner. Validation of the large-signal klystron Simulation code TESLA. IEEE Transactions on Plasma Science, 32(3):11361146, June 2004. Conference Name: IEEE Transactions on Plasma Science.

[18] Nicolas Crouseilles, Lukas Einkemmer, and Erwan Faou. Hamiltonian splitting for the vlasov-maxwell equations. Journal of Computational Physics, 283, 022015 .

[19] J.W. Eastwood. The virtual particle electromagnetic particle-mesh method. Computer Physics Communications, 64(2):252-266, 1991. 
[20] Alexandre Ern and Jean-Luc Guermond. Theory and Practice of Finite Elements. Applied Mathematical Sciences. Springer-Verlag, New York, 2004.

[21] M Esirkepov and T Zh. Exact charge-conservation scheme for particle-incell simulation with an arbitrary form factor. Computer Physics Communications, 2001.

[22] Dong-Ping Gao, Chao-Hai Du, Yao-Gen Ding, and Pu-Kun Liu. Development of a 2.5-Dimensional Particle-In-Cell Code for Efficient High-Power Klystron Design. IEEE Transactions on Plasma Science, 38(6):1277-1284, June 2010. Conference Name: IEEE Transactions on Plasma Science.

[23] Marc Gerritsma. An Introduction to a Compatible Spectral Discretization Method. Mechanics of Advanced Materials and Structures - MECH ADV MATER STRUCT, 19, January 2012.

[24] Vivette Girault and Pierre-Arnaud Raviart. Finite Element Methods for Navier-Stokes Equations: Theory and Algorithms. Springer Series in Computational Mathematics. Springer-Verlag, Berlin Heidelberg, 1986.

[25] Paul W. Gross and P. Robert Kotiuga. Electromagnetic theory and computation: a topological approach, volume 48 of Mathematical Sciences Research Institute Publications. Cambridge University Press, Cambridge, Cambridge, 2004.

[26] R. Hiptmair. Finite elements in computational electromagnetism. Acta Numerica, 11:237-339, 2002.

[27] R.W. Hockney and J.W. Eastwood. Computer simulation using particles. Taylor \& Francis, Inc, Bristol, PA, USA, 1988.

[28] Katharina Kormann and E. Sonnendrücker. Energy-conserving time propagation for a geometric particle-in-cell Vlasov-Maxwell solver. arXiv, October 2019.

[29] Michael Kraus, Katharina Kormann, Philip J. Morrison, and Eric Sonnendrücker. GEMPIC: geometric electromagnetic particle-in-cell methods. Journal of Plasma Physics, 83(4), August 2017.

[30] A. Bruce Langdon. On enforcing Gauss' law in electromagnetic particle-incell codes. Computer Physics Communications, 70(3):447-450, July 1992.

[31] Giovanni Lapenta. Exactly energy conserving semi-implicit particle in cell formulation. Journal of Computational Physics, 334:349-366, April 2017.

[32] Barry Marder. A method for incorporating Gauss' law into electromagnetic PIC codes. Journal of Computational Physics, 68(1):48-55, January 1987.

[33] Stefano Markidis and Giovanni Lapenta. The energy conserving particlein-cell method. J. Comput. Phys., 230(18):7037-7052, 2011. 
[34] Peter Monk. An analysis of Nédélec's method for the spatial discretization of Maxwell's equations. Journal of Computational and Applied Mathematics, 47(1):101-121, 1993.

[35] Peter Monk. Finite Element Methods for Maxwell's Equations. Oxford University Press, April 2003. Publication Title: Finite Element Methods for Maxwell's Equations.

[36] Haksu Moon, Fernando L Teixeira, and Yuri A Omelchenko. Exact chargeconserving scatter-gather algorithm for particle-in-cell simulations on unstructured grids: a geometric perspective. Computer Physics Communications, 194:43-53, 2015.

[37] C.-D. Munz, P. Omnes, R. Schneider, E. Sonnendrücker, and U Voß. Divergence Correction Techniques for Maxwell Solvers Based on a Hyperbolic Model. Journal of Computational Physics, 161(2):484-511, July 2000.

[38] D Y Na, Y A Omelchenko, H Moon, BHV Borges, and Fernando L Teixeira. Axisymmetric charge-conservative electromagnetic particle simulation algorithm on unstructured grids: Application to microwave vacuum electronic devices. J. Comput. Physics, 2017.

[39] Artur Palha, Pedro Pinto Rebelo, René Hiemstra, Jasper Kreeft, and Marc Gerritsma. Physics-compatible discretization techniques on single and dual grids, with application to the Poisson equation of volume forms. Journal of Computational Physics, 257:1394-1422, January 2014.

[40] Benedikt Perse. Energy-conserving Implicit Time Discretisation for the GEMPIC Framework. PhD thesis, TUM, 2017.

[41] Ahmed Ratnani and Eric Sonnendrücker. An Arbitrary High-Order Spline Finite Element Solver for the Time Domain Maxwell Equations. Journal of Scientific Computing, 51(1):87-106, April 2012.

[42] Andreas Stock, Jonathan Neudorfer, Rudolf Schneider, Christoph Altmann, and Claus-Dieter Munz. Investigation of the purely hyperbolic maxwell system for divergence cleaning in discontinuous galerkin based particle-in-cell methods. In COUPLED PROBLEMS 2011 IV International Conference on Computational Methods for Coupled Problems in Science and Engineering, 2011.

[43] Mark L Stowell and D A White. Discretizing Transient Current Densities in the Maxwell Equations. In ICAP 2009, 2009.

[44] Allen Taflove. Computational electrodynamics : the finite-difference timedomain method. Boston : Artech House, 1995.

[45] T Umeda, Y Omura, T Tominaga, and H Matsumoto. A new charge conservation method in electromagnetic particle-in-cell simulations. Computer Physics Communications, 156(1):73-85, December 2003. 
[46] John Villasenor and Oscar Buneman. Rigorous charge conservation for local electromagnetic field solvers. Computer Physics Communications, 69(2-3):306-316, March 1992.

[47] Erich S Weibel. Spontaneously Growing Transverse Waves in a Plasma Due to an Anisotropic Velocity Distribution. Physical Review Letters, 2(3):8384, February 1959. 